\title{
38. PALEOGENE AND EARLY NEOGENE OCEANOGRAPHY OF THE SOUTHERN INDIAN OCEAN: LEG 119 FORAMINIFER STABLE ISOTOPE RESULTS ${ }^{1}$
}

\author{
Enriqueta Barrera² and Brian T. Huber ${ }^{3}$
}

\begin{abstract}
Oxygen and carbon isotopic records of monogeneric and monospecific benthic and planktonic foraminifer samples from Sites 744 and 738 drilled on the southern end of the Kerguelen Plateau during ODP Leg 119 reveal the evolution of polar Indian Ocean water masses from the early Paleocene to the middle Miocene. Results from Site 738 are from sediments of early Paleocene to late Eocene age and those from Site 744 are late Eocene to middle Miocene. They suggest that intermediate waters at this location did not originate in the high latitudes during the early Eocene. Surface and near-surface waters cooled gradually after the maximum warming at $56 \mathrm{Ma}$, when surface waters were about $18^{\circ} \mathrm{C}$. Intermediate waters cooled after $52 \mathrm{Ma}$. The highest temperatures (lowest $\delta^{18} \mathrm{O}$ values) of the Cenozoic occurred from 56 to $52 \mathrm{Ma}$. The records of equatorial Pacific Site 577 and Weddell Sea Site 690 resemble that of the polar Indian Ocean in this interval. The well-documented $\delta^{13} \mathrm{C}$ excursions toward positive values in the late Paleocene and negative values in the early Eocene are represented by foraminifers increases of $1.5 \%$ and following decreases of about $3 \%$.

Most of the cooling in the Paleogene occurred in the middle and late Eocene. A $2{ }^{\circ} \mathrm{C}$ decrease of surface water at about 38.4 Ma heralded the beginning of extensive glacial conditions in Antarctica in the early Oligocene. At Site 744, the global $\delta^{18} \mathrm{O}$ shift just above the Eocene/Oligocene boundary is $1.15 \%$, and occurred gradually in sediments dated at 36.5-35.9 Ma. Ice-rafted debris was deposited beginning at 36.1 Ma for about the next $2 \mathrm{~m}$.y. This simultaneous occurrence of the global $\delta^{18} \mathrm{O}$ shift with ice-rafted debris is evidence for early Oligocene glaciation in East Antarctica. Moreover, early and late Oligocene Cibicidoides $\delta^{18} \mathrm{O}$ values between 2 and $2.2 \% 0$ indicate intermediate water cooling and a small ice-volume effect.

Production of cold dense bottom water in Antarctica was intensified with continental cooling and glaciation in the early Oligocene. Comparison of Oligocene and early Miocene isotopic data from high-latitude and low-latitude deepsea sites indicates that there were probably at least two sources of bottom waters at this time.
\end{abstract}

\section{INTRODUCTION}

One of the objectives of Ocean Drilling Program (ODP) Leg 119 was to document the regional climate and oceanography of polar waters in the southern Indian Ocean near Antarctica during the Tertiary. Sediments containing well-preserved foraminifers of Paleogene and early Neogene age were drilled at two sites on the southern end of the Kerguelen Plateau (Fig. 1). At Site $738\left(62^{\circ} 42.54^{\prime} \mathrm{S}, 82^{\circ} 47.25^{\prime} \mathrm{E}\right.$; water depth $\left.2253 \mathrm{~m}\right)$ a sequence of Turonian to Quaternary sediments was recovered while the sedimentary record at Site $744\left(61^{\circ} 34.66^{\prime} \mathrm{S}, 80^{\circ} 35.46^{\prime} \mathrm{E}\right.$; water depth $2317 \mathrm{~m}$ ) spans the late Eocene to the late Miocene (Fig. 2 ). The high-latitude locations and the proximity to the margins of East Antarctica (Fig. 1) make these sites ideal monitoring stations of climatic events and associated oceanographic changes within surface to intermediate water depths in the surrounding Antarctic Ocean. Site 744 also contains an expanded section of the Eocene/Oligocene transition with ice-rafted debris that provides the means to study events associated with the onset of Oligocene glaciation in Antarctica in great detail. We report the results of oxygen and carbon isotope analyses of monogeneric and monospecific benthic and planktonic foraminifers of Paleogene to late Miocene age from these southernmost Indian Ocean sites. Paleodepths have been estimated, using normal subsidence rates for oceanic crust (Detrick et al., 1977) as follows: $1,000 \mathrm{~m}$ in the earliest Paleocene; 1,350 in the early Eocene; 1,800 in the

\footnotetext{
${ }^{1}$ Barron, J., Larsen, B., et al., 1991. Proc. ODP, Sci. Results, 119: College Station, TX (Ocean Drilling Program).

2 Department of Geological Sciences, University of Michigan, Ann Arbor, MI 48109-106, U.S.A.

3 Department of Paleobiology, NHB-121, National Museum of Natural History, Smithsonian Institution, Washington, D.C. 20560, U.S.A.
}

earliest Oligocene; and approximately $2,050 \mathrm{~m}$ in the earliest early Miocene. Based on comparison of isotopic compositions of benthic and planktonic foraminifers, we draw conclusions regarding the response of the polar water column (at surface to intermediate water depths) to climatic changes in the high southern latitudes, and infer sources of the intermediate waters.

At present, these sites on the southern end of Kerguelen Plateau are located between the Polar Front, which is more than $1500 \mathrm{~km}$ to the north, and the Antarctic Divergence at about $65^{\circ} \mathrm{S}$ (Fig. 1). The Polar Front separates the cold Antarctic surface waters from the warmer Subantarctic surface waters. The Antarctic Divergence separates the Antarctic Coastal Current flowing west along the Antarctic margins from the Antarctic Circumpolar Current (ACC) flowing east in a zone north of the Antarctic Coastal Current. Site 738 is beneath the southern part of the Antarctic Circumpolar Current. The southern Kerguelen Plateau rises 2-4 km above the adjacent ocean basins to the east and west, and more than $3000 \mathrm{~m}$ above the narrow passage to the south that separates it from Antarctica (Fig. 1). The cold and very dense Antarctic Bottom Water (AABW) flows along this passage.

These oceanographic features of the Antarctic ocean developed as a response to changes in tectonic configurations and climate of Antarctica. Evidence from oxygen isotopic studies, and floral and faunal studies has revealed that early Paleogene polar temperatures were significantly warmer than today (Shackleton and Kennett, 1975; Savin et al., 1975; Kennett and Stott, 1990; Axelrod, 1984; Kemp, 1978). Warmer polar temperatures imply that oceanographic processes and the structure of the water column in the southern high latitudes were also different. Surface water temperatures declined during the second half of the $\mathrm{Pa}$ leogene, and colder conditions more similar to the present ones were established in the Oligocene (Shackleton and Kennett, 1975; Kennett and Shackleton, 1976; Kennett, 1977; and others). In 


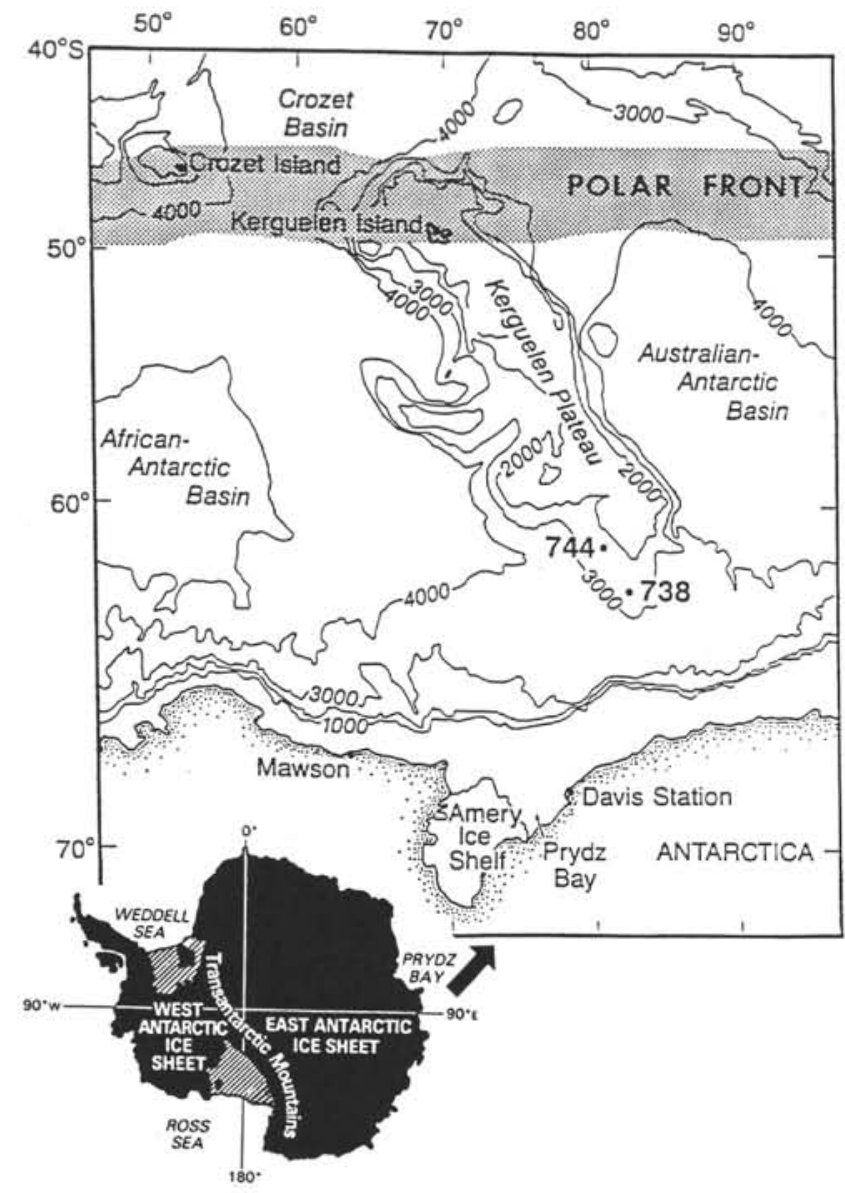

Figure 1. Locations of ODP Sites 738 and 744 on the southern Kerguelen Plateau and in relation to the Polar Front Zone. Contours indicate bathymetry in meters.

the early Oligocene, continental glaciation developed, and cold dense bottom waters produced during sea ice formation began to originate in Antarctic coastal regions (Kennett and Shackleton, 1976; Kennett, 1977; Barrett, 1989;).

It has been suggested that prior to high-latitude cooling, bottom waters formed by the sinking of warm saline plumes produced by evaporation in low- and mid-latitude shelf areas (Chamberlain, 1906; Brass et al. 1982). Recently, Kennett and Stott (1990) have suggested that deep waters in the southern South Atlantic during most of the Eocene originated in the low latitudes. According to Kennett and Stott (1990), intermediate waters were of Antarctic origin. Woodruff and Savin (1989) concluded that early Miocene intermediate waters in the Indian Ocean were warm and saline derived from a Tethyan source. The recovery of Paleocene to Eocene foraminifer sediments at Site 738 provides the opportunity to investigate isotopically if intermediate waters in the southern Indian Ocean were formed on the Antarctic margins. Moreover, we also examine the nature and sequence of oceanographic events near the Eocene/Oligocene boundary when the presence of significant glaciation on Antarctica has been inferred (Keigwin and Keller, 1984; Miller and Thomas, 1985; Keigwin and Corliss, 1986). Ice-rafted debris associated with the global $\delta^{18} \mathrm{O}$ shift of about $1 \%$ recognized at Site 744 just above this transition represents direct evidence for the build-up of significant continental ice in the early Oligocene.

\section{PREVIOUS ISOTOPIC STUDIES}

Pioneering paleoclimatic studies by Emiliani (1956) documented the warm polar climate of the early Tertiary from benthic foraminifer ${ }^{18} \mathrm{O} /{ }^{16} \mathrm{O}$ ratios in deep-sea sediments. This was based on the idea that oxygen isotopic compositions of deep-sea benthic foraminifers reflect temperatures in the high latitudes where bottom waters form (Emiliani, 1956). Yet, the early Tertiary climate and paleoceanography of the high latitudes have not been isotopically studied in detail. Isotopic records for Antarctic waters have been generated for sediments recovered during Leg 29 in the southwestern Pacific Ocean (Shackleton and Kennett, 1975), and more recently for Weddell Sea sediments drilled during Leg 113 (Kennett and Stott, 1990; Stott et al., 1990). Antarctic climates have more commonly been inferred from analyses of benthic foraminifers from lower-latitude deepsea sediments.

A global increase in $\delta^{18} \mathrm{O}$ values of benthic foraminifer from low- and mid-latitude sites was recorded in lower Paleocene sediments reaching a maximum near the Foraminiferal Zone P3/P4 boundary at about $60 \mathrm{Ma}$ (Oberhansli and Toumarkine, 1985; Shackleton, 1986; Miller et al., 1987a; Kennett and Stott, 1990). Prior to this study, there were no isotopic data of early Paleocene planktonic foraminifers from high-latitude sites, but faunal and floral evidence from mid- and high-latitude locations indicate cooling occurred from the early to the late Paleocene and that warming occurred subsequently at the Paleocene/Eocene boundary (Haq et al., 1977; Wolfe, 1978). The increase in high-latitude temperatures from the late Paleocene to the early Eocene was reflected in a global decrease in deep-sea benthic foraminifer $\delta^{18} \mathrm{O}$ of about $1.5 \%$ reaching a minimum between about 56 and 54 Ma values (Shackleton et al., 1984; Miller et al., 1987b; Kennett and Stott, 1990; Katz and Miller, 1991; and others). However, most of the isotopic change occurred near the Paleocene/Eocene boundary (Shackleton, 1986). If this $1.5 \%$ change were entirely due to temperature, then deep waters would have warmed by about $6^{\circ} \mathrm{C}$ to reach temperatures close to $14^{\circ} \mathrm{C}$ (Shackleton and Kennett, 1975; Shackleton et al., 1984; Shackleton, 1986; Miller et al., 1987b). Paleotemperature estimates of early Eocene surface waters near Antarctica are close to $18^{\circ} \mathrm{C}$ based on analysis of planktonic foraminifers (Shackleton and Kennett, 1975; Stott and Kennett, 1990). With the warming of the poles, the latitudinal thermal gradient was severely reduced at this time (Shackleton and Boersma, 1981).

Subsequent to the early Eocene $\delta^{18} \mathrm{O}$ minimum, benthic foraminifer and high- and mid-latitude planktonic $\delta^{18} \mathrm{O}$ values increased by about $2 \%$ during the middle and late Eocene (Shackleton and Kennett, 1975; Oberhansli et al., 1984; Shackleton et al., 1984; Kennett and Stott, 1990; and others). A marked $\delta^{18} \mathrm{O}$ increase of about $1 \% 0$ was observed in lowermost middle Eocene sediments (Shackleton et al., 1984; Shackleton, 1986; Oberhansli, 1986; Miller et al., 1987a). The beginning of this event was correlated to Magnetochron C22 at about 52.6-52 Ma and ending in Magnetochron C21 at about $49 \mathrm{Ma}$ (Katz and Miller, 1991). A $\delta^{18} \mathrm{O}$ increase of lesser magnitude was reported near the middle/ late Eocene boundary (Oberhansli et al., 1984; Oberhansli and Toumarkine, 1985; Keigwin and Corliss 1986). In the latest late Eocene, this trend of increasing $\delta^{18} \mathrm{O}$ was punctuated by a small decrease in benthic foraminifer and mid- and high-latitude planktonic foraminifer values (Savin et al., 1975; Shackleton and Kennett, 1975; Shackleton et al., 1984; and others).

The largest Paleogene increase in benthic $\delta^{18} \mathrm{O}$ values (1$1.5 \%$ ) was recorded just above the Eocene/Oligocene boundary at the base of Chron $13 \mathrm{~N}$ with an estimated age of $35.9 \mathrm{Ma}$ (Oberhansli et al., 1984; Miller et al., 1988). Unlike earlier Eocene increases in benthic $\delta^{18} \mathrm{O}$ values which have been attributed 
to declines in temperature, the early Oligocene ${ }^{18} \mathrm{O}$ enrichment has been interpreted as a combination of both high-latitude cooling and increased ice mass in Antarctica (Matthews and Poore, 1980; Keigwin and Keller, 1984; Keigwin and Corliss, 1986; Miller et al., 1987a). Two arguments have been proposed for ice-volume in the earliest Oligocene. (1) Concomitant with the ${ }^{18} \mathrm{O}$ enrichment of benthic foraminifers, $\delta^{18} \mathrm{O}$ of planktonic foraminifers globally increased by a smaller amount (Matthews and Poore, 1980; Keigwin and Keller, 1984; Poore and Matthews, 1984; Keigwin and Corliss, 1986). Based on the assumption that surface water temperatures in the tropics have remained constant, this global ${ }^{18} \mathrm{O}$ enrichment of seawater must reflect increased ice volume (Matthews and Poore, 1980; Poore and Matthews, 1984; Keigwin and Corliss, 1986). (2) Calculated paleotemperatures of deep waters in the early Oligocene, based on the assumption that the world had no significant mass of ice to affect seawater $\delta^{18} \mathrm{O}$, were as low or lower than present temperatures (Keigwin and Keller, 1984; Miller and Thomas, 1985, Miller et al., 1987a). Such low temperatures of deep water are inconsistent with the idea that polar temperatures in the early Oligocene were warmer than at present (Keigwin and Keller, 1984). Based on similar arguments, the global ${ }^{18} \mathrm{O}$ enrichment in benthic foraminifers near the early/late Oligocene boundary is inferred to reflect the effect of ice volume on sea water $\delta^{18} \mathrm{O}$ and decreasing deep-water temperatures (Keigwin and Keller, 1984; Miller and Thomas, 1985). Recent sedimentologic evidence points to extensive Oligocene glaciation in Antarctica (Barrett, 1989). Lower Oligocene glaciomarine sediments and upper Oligocene diamictites interbedded with glaciomarine sediments drilled in the southwestern Ross Sea indicate glacial conditions followed by intermittent grounding of a continental ice sheet at sea level during the late Oligocene (Barrett, 1989).

The most remarkable features of the global Paleogene carbon isotopic record are the high planktonic and benthic foraminifer $\delta^{13} \mathrm{C}$ values in the late Paleocene between about 61 and $58 \mathrm{Ma}$ (Oberhansli and Toumarkine, 1985) and the subsequent sharp decline in values in lower Eocene sediments (Shackleton and Hall, 1984). The later event occurred in sediments correlated with magnetozones $\mathrm{C} 24 \mathrm{R}$ to $\mathrm{C} 24 \mathrm{~N}$ with age estimates between about 58.5 and $56 \mathrm{Ma}$ (Miller et al., 1987b). These $\delta^{13} \mathrm{C}$ events occurred while global temperatures were increasing. Constancy in the surface-to-bottom $\delta^{13} \mathrm{C}$ gradient (Miller, et al., $1987 \mathrm{~b}$ ) indicates that $\delta^{13} \mathrm{C}$ changes were not caused by changes in productivity as proposed by some authors (Shackleton and Hall, 1984; Shackleton 1987). These $\delta^{13} \mathrm{C}$ and $\delta^{18} \mathrm{O}$ fluctuations may be linked to the extensive volcanism near the Paleocene/ Eocene boundary which may have increased carbon dioxide levels in the atmosphere and caused global warming (Owens and Rea, 1985).

Indian Ocean stable isotope records of Paleogene age were generated by Oberhansli (1986) for Deep Sea Drilling Project (DSDP) Sites 217, 237, 220, and 253. The early Paleogene records are based on analysis of bulk carbonate, whereas the Oligocene record is of monospecific foraminifers. In general, the Paleogene Indian Ocean patterns of $\delta^{18} \mathrm{O}$ and $\delta^{13} \mathrm{C}$ variations are similar to global patterns discussed above. Ours, however, is the first comprehensive study of the Paleogene Indian Ocean based on analyses of foraminifer samples.

In the following sections, we present the early Paleocene to middle Eocene records and results suggesting that the source of intermediate waters in the early Eocene was in the low latitudes. Then, we present late Eocene to Miocene records, and discuss the results of isotopic and sedimentological studies of the Eocene/Oligocene transition, and implications of Antarctic glaciation for deep water origin and circulation.

\section{MATERIAL AND METHODS}

\section{Samples}

Samples from Site 738 and 744 that were analyzed in this study are listed in Tables 1-4. Foraminifer samples from Site 738 consist of the planktonic species Chiloguembelina spp., Acarinina primitiva, Globigerinatheka index, Subbotina spp., Subbotina linaperta, Subbotina angiporoides, and Globorotaloides suteri and the benthic taxa Gavelinella beccariiformis, Nuttallides truempyi, and Cibicidoides spp. At Site 744, isotopic analyses were performed in the planktonic species Chiloguembelina cubensis and Globorotaloides suteri, and the benthic taxa Nuttallides spp., Stilostomella subspinosa, and Cibicidoides spp. These taxa were selected based on: (1) their abundance in sediment samples, (2) their stratigraphic range, and (3) because their isotopic compositions are inferred to reflect conditions at different depths in the water column. Isotopic results of Stilostomella subspinosa and Nuttallides spp. are not included in our discussion of isotopic time series because of the uncertainty regarding their isotopic fractionations and/or the limited data set.

Late Eocene and younger foraminifer specimens were deemed well preserved based on Scanning Electron Microscope (SEM) observations of broken surfaces and interior surfaces of tests. Where present, overgrowth crystals on interior surface chambers were small and volumetrically insignificant (see Barrera et al., this volume). We did not conduct a thorough study of the preservation of foraminifer calcite of older samples. SEM observations were made of a few selected specimens at various intervals and overgrowth crystals were noted, in general increasing in size with increasing age. The wall structure of some Paleocene and early Eocene specimens show evidence of replacement by secondary calcite. In general, we expect $\delta^{13} \mathrm{C}$ values of foraminifers in deep-sea sediments with low organic matter to be less affected by dissolution-reprecipitation because the carbon reservoir in pore waters is significantly smaller than in the carbonate. Oxygen isotopic compositions can be significantly modified by dissolution-reprecipitation processes if exchange occurred (1) at high temperatures, and/or (2) with interstitial waters depleted in ${ }^{18} \mathrm{O}$ from other diagenetic reactions in the sediments (Garrison, 1981). Burial depths at Site 738 are less than $400 \mathrm{~m}$, and in situ temperatures measured in the upper $200 \mathrm{~m}$ of section near Site 738 range between $-2^{\circ}$ and $+2^{\circ} \mathrm{C}$ (Barron, Larsen, et al., 1989). No analyses of pore water $\delta^{18} \mathrm{O}$ values are available. Although we cannot rule out the possibility that isotopic signatures of Paleocene to middle Eocene foraminifer tests partially replaced by diagenetic calcite have been modified, the similarity of absolute $\delta^{18} \mathrm{O}$ values and trends to those observed at other deep-sea sites, as subsequently discussed, suggests that measured values are not very different from original foraminifer values.

\section{Analytical Methods}

Samples were disaggregated by stirring in water heated to $40^{\circ}-50^{\circ} \mathrm{C}$ and ultrasonically cleaned in tap water before sieving them through a $63 \mu \mathrm{m}$ screen. Most planktonic foraminifer samples were picked from the $150-250 \mu \mathrm{m}$ size fraction. Specimens of Chiloguembelina cubensis were taken from the larger-than $125 \mu \mathrm{m}$ fraction. Benthic foraminifers were picked from the larger-than $250 \mu \mathrm{m}$ fraction. Prior to isotopic analysis, foraminifer specimens were ultrasonically agitated in distilled water to remove adhering particles.

Isotopic analyses were performed at the Stable Isotope Laboratory of the University of Michigan, Ann Arbor, MI. Analyses are reported in $\delta$ notation as per mil deviations from the Peedee Belemnite (PDB) standard. Samples were reacted with anhydrous phosphoric acid at $55^{\circ} \mathrm{C}$ in an on-line gas extraction line 
Table 1. Oxygen and carbon isotope ratios of foraminifer taxa from Hole 744B.

\begin{tabular}{|c|c|c|c|c|c|c|c|c|}
\hline \multirow{4}{*}{$\begin{array}{l}\text { Core, section } \\
\text { interval (cm) }\end{array}$} & \multirow[t]{4}{*}{$\begin{array}{l}\text { Depth } \\
\text { (mbsf) }\end{array}$} & \multirow[t]{4}{*}{$\begin{array}{l}\text { Nanno } \\
\text { biozone }\end{array}$} & \multirow[t]{4}{*}{$\begin{array}{l}\text { Ant. foram } \\
\text { biozone }\end{array}$} & \multirow{4}{*}{$\begin{array}{l}\text { Age } \\
\text { (m.y.) }\end{array}$} & \multicolumn{4}{|c|}{ SELECTED FORAMINIFER } \\
\hline & & & & & \multicolumn{2}{|c|}{ Cibicidoides } & \multicolumn{2}{|c|}{ Nuttallides } \\
\hline & & & & & \multicolumn{2}{|l|}{ spp. } & \multicolumn{2}{|l|}{ spp. } \\
\hline & & & & & 8180 & $\delta 13 c$ & 8180 & $\delta 13 C$ \\
\hline \multicolumn{9}{|l|}{$119-7448$} \\
\hline $4 \mathrm{H}-5,60-62$ & 28.11 & CN5b-CN10 & Unzoned & 9.27 & 2.75 & 0.94 & 2.56 & 0.75 \\
\hline $4 \mathrm{H}-6,60-62$ & 29.61 & CN5b-CN 10 & Unzoned & 9.41 & 2.72 & 0.82 & & \\
\hline $5 H-4,60-62$ & 36.11 & CN5b-CN10 & Unzoned & 10.04 & & & 2.77 & 0.86 \\
\hline $5 \mathrm{H}-5,60-62$ & 37.61 & CN5b-CN10 & Unzoned & 10.19 & 2.78 & 1.26 & & \\
\hline $5 \mathrm{H}-7,60-62$ & 40.61 & CN5b-CN10 & Unzoned & 10.47 & 2.90 & 1.24 & 2.46 & -0.05 \\
\hline $6 \mathrm{H}-1,60-62$ & 41.11 & CN5b-CN10 & Unzoned & 10.52 & & & 2.26 & 0.73 \\
\hline $6 \mathrm{H}-4,60-62$ & 45.61 & CN5B-CN10 & Unzoned & 10.96 & 2.51 & 1.36 & & \\
\hline $6 \mathrm{H}-5,70-72$ & 47.11 & CN5b-CN10 & Unzoned & 11.11 & & & 2.91 & 0.47 \\
\hline $7 \mathrm{H}-2,70-72$ & 52.21 & CN5 - - CN10 & Unzoned & 11.60 & & & 2.59 & 0.86 \\
\hline $7 \mathrm{H}-3,70-72$ & 53.71 & CN5b-CN10 & Unzoned & 12.40 & & & & \\
\hline $7 H-4,70-72$ & 55.21 & CN1-CN5a & Unzoned & 13.69 & 1.71 & 1.38 & 1.88 & 1.19 \\
\hline $7 H-5,70-72$ & 56.71 & CN1-CN5a & Unzoned & 14.00 & 1.47 & 1.46 & 1.62 & 1.27 \\
\hline $7 \mathrm{H}-6,70-72$ & 58.21 & CN1-CN5a & Unzoned & 14.20 & 1.85 & 1.79 & & \\
\hline $7 \mathrm{H}-7,70-72$ & 59.71 & CN1-CN5a & Unzoned & 14.80 & & & & \\
\hline $8 H-1,95-100$ & 60.11 & CN1-CN5a & Unzoned & 14.90 & & & 1.10 & 1.41 \\
\hline $8 \mathrm{H}-2,95-100$ & 61.61 & CN1-CN5a & Unzoned & 15.30 & & & & \\
\hline $8 H-3,95-100$ & 63.11 & CN1-CN5a & Unzoned & 15.70 & 1.78 & 1.76 & & \\
\hline $8 H-4,95-100$ & 64.61 & CN1-CN5a & Unzoned & 16.15 & 1.53 & 1.05 & & \\
\hline $8 \mathrm{H}-5,60-62$ & 66.11 & CN1-CN5a & Unzoned & 16.60 & 2.01 & 1.51 & 1.46 & 1.60 \\
\hline $8 H-6,60-62$ & 67.61 & CN1-CN5a & Unzoned & 17.20 & 1.51 & 1.71 & & \\
\hline $9 \mathrm{H}-1,73-78$ & 69.59 & CN1-CN5a & Unzoned & 17.30 & & & & \\
\hline $9 \mathrm{H}-3,58-60$ & 72.59 & CN1-CN5a & Unzoned & 17.50 & 1.87 & 1.44 & 1.83 & 1.28 \\
\hline $9 H-4,58-60$ & 74.09 & CN1-CN5a & Unzoned & 17.80 & 1.95 & 1.54 & & \\
\hline $9 \mathrm{H}-5,58-60$ & 75.59 & CN1-CN5a & Unzoned & 17.95 & 1.76 & 1.25 & & \\
\hline $9 \mathrm{H}-6,58-60$ & 77.09 & CN1-CN5a & Unzoned & 18.10 & 1.97 & 1.40 & 1.79 & 1.07 \\
\hline
\end{tabular}

coupled to the inlet of a VG602E mass spectrometer, or were reacted individually at $70^{\circ} \mathrm{C}$ in a Kiel device and analyzed in a Finnigan 261 mass spectrometer. Compositions of isotopic measurements made relative to standard $\mathrm{CO}_{2}$ gas are related to PDB values through numerous analyses of National Bureau of Standards Reference Material \#20 (Solnhofen limestone), which is taken to have a $\delta^{18} \mathrm{O}$ value of $-4.14 \%$ and a $\delta^{13} \mathrm{C}$ value of $-1.06 \%$ (Craig, 1957). Standard replicates have a precision of $\pm 0.05 \%$ for $\delta^{18} \mathrm{O}$ and $\delta^{13} \mathrm{C}$. Water temperatures in Paleogene oceans were calculated using the equation of O'Neil et al. (1969) as recast by Shackleton (1974) and assuming that the oxygen isotopic composition of the oceans was $-1 \%$ relative to Standard Mean Ocean Water (SMOW) before the growth of the ice cap.

\section{STRATIGRAPHY AND LITHOLOGY}

Age determinations of sediments at Site 744 are based on the integration of calcareous and siliceous plankton biostratigraphic data with magnetic polarity data and ${ }^{87} \mathrm{Sr} /{ }^{86} \mathrm{Sr}$ isotope stratigraphy (Barron et al., this volume; Barrera et al, this volume) using the chronology of Berggren et al. (1985a, 1985b). Calcareous datums (Wei and Wise, this volume; Huber, this volume) tied to the chronology of Berggren et al. (1985b) were used to assign ages to sediments at Site 738 (Barron et al., this volume). Cal- careous datums were also calibrated with the magnetic polarity stratigraphy of lower Paleocene sediments at Site 738 (see Huber, this volume). Because of poor reçovery in the upper Paleocene to lower middle Eocene section at Hole $738 \mathrm{C}$, absolute age assignments are uncertain.

Lower Paleocene to lower Eocene sediments at Site 738 are calcareous chalks with relatively minor amounts of foraminifer and nannofossils and numerous chert nodules. As in other deepsea sections of this age, recovery was not continuous because of the high abundance of chert layers in this interval and the partial silicification of the chalk by opal-CT and quartz. Nevertheless, the isotopic record for this interval, as discussed in a subsequent section, is fairly detailed. The lower to middle Eocene section is a calcareous nannofossil ooze that grades downcore into chalk. Layers of porcellanite chert and concretions are also found in this interval. The middle Eocene to lower Oligocene sequence is a calcareous nannofossil ooze. At Site 744, a similar lithology characterizes the section from the upper Eocene to upper Miocene, where foraminifers constitute a few percent of the sediment.

\section{FORAMINIFER ISOTOPIC SYSTEMATICS}

Most Holocene deep-water benthic foraminifer species tests are in neither oxygen nor carbon isotopic equilibrium with am- 
bient seawater (Shackleton, 1974; Woodruff et al., 1980; Belanger et al., 1981; and others). Interspecific differences in oxygen and carbon fractionation of well-preserved older benthic foraminifers appear to remain about the same through time, implying that species departure from equilibrium are approximately constant (Savin et al., 1981; Shackleton et al., 1984). Estimates of depletion in ${ }^{18} \mathrm{O}$ relative to equilibrium values by species of Cibicidoides (the taxa most commonly used in isotopic paleoceanographic studies) ranges from 0.5 to $0.7 \%$ (Woodruff et al., 1980; Belanger et al., 1981; Savin et al., 1981; Shackleton et al., 1984). $\delta^{13} \mathrm{C}$ values of Cibicidoides are similar to those of dissolved bicarbonate in ambient seawater (Belanger et al., 1981; Graham et al., 1981; Shackleton et al., 1984). Calculated isotopic differences between the different taxa analyzed in this study are listed in Table 5. Standard deviations of these differences are high resulting from fair reproducibility of some of the data. Based on these differences and the magnitude of the reported departure of Cibicidoides spp. from equilibrium precipitation (Shackleton et al., 1984), adjustments listed in Table 6 were made to the benthic foraminifer data in two of the figures, as noted in the text. No adjustments were made to the benthic $\delta^{13} \mathrm{C}$ data in our figures. Although previous studies have reported that $\delta^{13} \mathrm{C}$ values of these taxa are similar to those of dissolved bicarbonate (Shackleton et al., 1984), we have noted that there are significant differences among $\delta^{13} \mathrm{C}$ values of the various taxa analyzed in this study (Table 5). Therefore, the same taxon is used in the comparison of benthic $\delta^{13} \mathrm{C}$ records between sites.

Carbon isotope values of Holocene planktonic foraminifers are in disequilibrium with ambient bicarbonate $\delta^{13} \mathrm{C}$ but are very close to that of total dissolved $\mathrm{CO}_{2}$ (Williams et al., 1977). Planktonic foraminifer ${ }^{18} \mathrm{O} /{ }^{16} \mathrm{O}$ ratios appear to be in or close to equilibrium (Williams et al., 1979). The ranking of planktonic $\delta^{13} \mathrm{C}$ and $\delta^{18} \mathrm{O}$ values is interpreted to represent the carbon isotopic composition and temperature of the water where the foraminifer calcified its test. The surface-dweller species should have the lowest $\delta^{18} \mathrm{O}$ and the highest $\delta^{13} \mathrm{C}$ values. These reflect the high temperatures of surface waters, and the nutrient depletion and/or high $\delta^{13} \mathrm{C}$ values of surface water from the removal of ${ }^{12} \mathrm{C}$ by plankton productivity. On this basis, Chiloguembelina cubensis and species of Acarinina have been determined to be surface-dwelling taxa (Keigwin and Corliss, 1986; Shackleton et al., 1985). Subbotinids, chiloguembelids, and globorotaloids usually measure the highest $\delta^{18} \mathrm{O}$ and lowest $\delta^{13} \mathrm{C}$ values indicating a subsurface habitat below the shallow-dwelling species, and probably below the thermocline (Shackleton et al., 1985; Boersma et al., 1987). At present, the seasonal temperature contrast of surface water is high in the high latitudes. In the Paleogene, it is possible that the shallow-water species may have only grown during the warmest part of the year or summer. The temperature of near-surface waters may have remained more or less constant throughout the year and thus be representative of surface temperatures during winter when the thermocline disappeared, as it does today. Isotopic differences among planktonic foraminifers can be interpreted either in terms of the vertical stratification of the water column or in terms of the seasonal temperature contrast.

\section{RESULTS}

Oxygen and carbon isotope results of benthic and planktonic foraminifers from Holes 744B, 744A, 738B. and 738C are tabulated in Tables 1-4. Adjustments to correct for estimated disequilibrium precipitation of benthic foraminifers are listed in Table 6 and have only been applied to the data plotted in Figures $3 \mathrm{~A}$ and 8 . Values discussed in the text have not been adjusted unless noted. In the discussion in the following sections, isotopic compositions of benthic foraminifer taxa are taken to re- flect conditions in the intermediate water mass in the southern Indian Ocean. Isotopic compositions of the planktonic foraminifer species Globorotaloides suteri and species of Subbotina and Paleocene and early Eocene chiloguembelinids reflect conditions of their inferred habitat in near-surface waters below the thermocline, whereas those of species of Acarinina and Chiloguembelina cubensis are indicative of conditions close to those of surface waters.

\section{Early Paleocene to Late Middle Eocene Records}

\section{Oxygen Isotope Results}

The $\delta^{18} \mathrm{O}$ values of $G$. beccariiformis and $N$. truempyi increased by about $1 \%$ in the early Paleocene to a maximum at 322.1 mbsf (Antarctic Foraminiferal Zone AP4) in lower upper Paleocene sediments (Fig. 2). Isotopic ratios $\left({ }^{18} \mathrm{O} /{ }^{16} \mathrm{O}\right.$ ) of these species at the late Paleocene maximum were about $0.5 \%$ (Fig. 2 ). Across the Paleocene/Eocene boundary, N. truempyi $\delta^{18} \mathrm{O}$ values decreased to a minimum of $-1 \%$ in lower Eocene sediments at 274.77 mbsf (Antarctic Foraminiferal Zone AP6a) with an estimated age of about 55.6 Ma (Fig 3A). At this time, intermediate waters reached temperatures close to $13^{\circ} \mathrm{C} . \delta^{18} \mathrm{O}$ values of early Eocene Cibicidoides spp. exhibit the same pattern as those of $N$. truempyi but were lowest $(-0.8 \%$ ) at $53.8 \mathrm{Ma}$ (Antarctic Foraminiferal Zone AP6b) (Figs. 2 and 3A). After about $51 \mathrm{Ma}$ (in Antarctic Foraminiferal Zone AP7), benthic foraminifer $\delta^{18} \mathrm{O}$ began a long-term trend of increasing values for the rest of the Paleogene (Figs 2 and $3 \mathrm{~A}$ ). Pronounced Eocene ${ }^{18} \mathrm{O}$ enrichments occurred from just above the early/middle Eocene boundary to the middle Eocene at about $47 \mathrm{Ma}$ (across the transition of Antarctic Foraminiferal Zone AP7/AP8) and across the middle to late Eocene boundary, where Cibicidoides spp. attained values close to $1 \%$.

Oxygen isotopic ratios of Subbotina spp. and Acarinina spp. varied little in the late Paleocene (Figs. 2 and 3A). Based on one data point, temperatures of high-latitude surface waters did not decreased in the early late Paleocene as those at of intermediate waters. Planktonic $\delta^{18} \mathrm{O}$ followed the same trend of the benthic taxa across the Paleocene/Eocene boundary reaching minimum values near 56.6 Ma (Antarctic Foraminiferal Zone AP6a). Afterward, the ${ }^{18} \mathrm{O}$ enrichment of the planktonic taxa was nearly continuous in lower and middle Eocene sediments.

\section{Carbon Isotope Results}

Carbon isotope ratios of $N$. truempyi and $G$. beccariiformis were low from the early to the early late Paleocene and subsequently increased rapidly by more than $1.5 \%$ in the late Paleocene to peak values greater than $2 \%$ (at about 59 to $60 \mathrm{Ma}$, 312.3 and 321.9 mbsf, respectively) (Figs. 2 and 3B). From the late Paleocene maximum, $N$. truempyi $\delta^{13} \mathrm{C}$ values declined to an early Eocene minimum close to $-0.5 \%$ at $55.6 \mathrm{Ma}(274.7$ mbsf) at the same time that the $\delta^{18} \mathrm{O}$ minimum was recorded. Subsequently, benthic foraminifer $\delta^{13} \mathrm{C}$ reached values close to $1 \%$ in the late early Eocene and remained at this level through the early middle Eocene.

$\delta^{13} \mathrm{C}$ values of the planktonic taxa varied similarly to benthic $\delta^{13} \mathrm{C}$ values in the Paleocene to early Eocene interval (Figs. 2 and $3 \mathrm{~B})$. After the early Eocene minimum, $\delta^{13} \mathrm{C}$ of Subbotina spp. increased gradually by about $0.5 \% 0$ and remained in the range of $1-1.3 \%$ in middle Eocene sediments. ${ }^{13} \mathrm{C} /{ }^{12} \mathrm{C}$ ratios of early and middle Eocene Acarinina are highly variable and do not appear to follow the same trend as near-surface and intermediate waters.

\section{Surface to Intermediate Water Gradients}

During the transition from the temperate Paleocene to the warm early Eocene, there was a change in the thermal stratifica- 
SELECTED FORAMINIFER TAXA

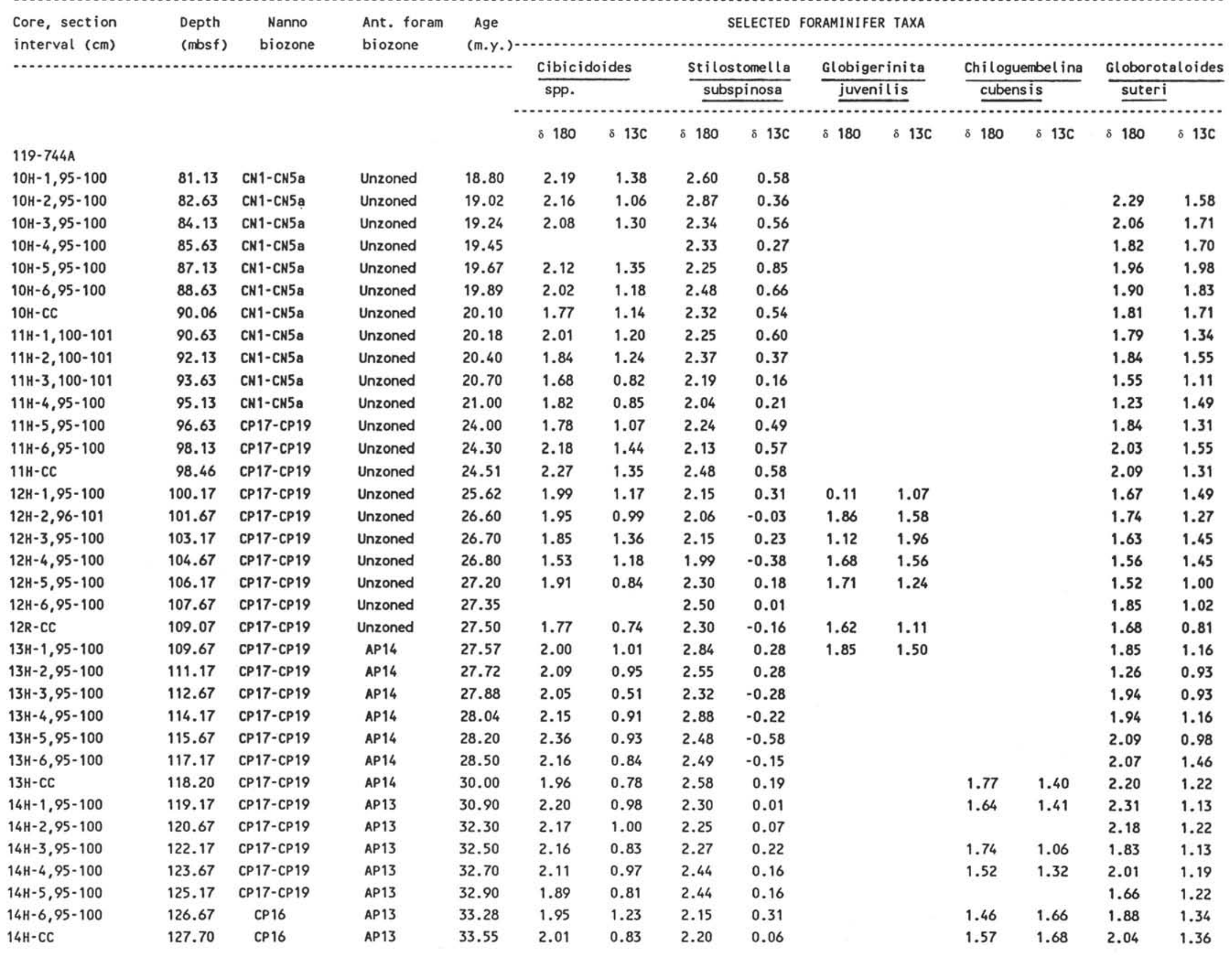




\begin{tabular}{|c|c|c|c|c|c|c|c|c|c|c|c|c|c|c|}
\hline \multirow{3}{*}{$\begin{array}{l}\text { Core, section } \\
\text { interval }(\mathrm{cm})\end{array}$} & \multirow{3}{*}{$\begin{array}{l}\text { Depth } \\
\text { (mbsf) }\end{array}$} & \multirow{3}{*}{$\begin{array}{c}\text { Nanno } \\
\text { biozone }\end{array}$} & \multirow{3}{*}{$\begin{array}{l}\text { Ant. foram } \\
\text { biozone }\end{array}$} & \multirow{3}{*}{$\begin{array}{l}\text { Age } \\
(\mathrm{m} . \mathrm{y} .)- \\
. . . .\end{array}$} & \multicolumn{6}{|c|}{ SELECTED FORAMINIFER TAXA } & & & . & \\
\hline & & & & & \multicolumn{2}{|c|}{$\frac{\text { Cibicidoides }}{\text { spp. }}$} & \multicolumn{2}{|c|}{$\frac{\text { Stilostomella }}{\text { subspinosa }}$} & \multicolumn{2}{|c|}{$\frac{\text { Globigerinita }}{\text { juvenilis }}$} & \multicolumn{2}{|c|}{$\frac{\text { Chiloguembelina }}{\text { cubensis }}$} & \multicolumn{2}{|c|}{$\frac{\text { Globorotaloides }}{\text { suteri }}$} \\
\hline & & & & & $\delta 180$ & $\delta 13 c$ & 8180 & $\delta 13 c$ & $\delta 180$ & $813 C$ & $\delta 180$ & $\delta 13 \mathrm{C}$ & $\delta 180$ & $813 c$ \\
\hline $15 H-1,95-100$ & 128.67 & CP16 & AP13 & 33.80 & 2.23 & 0.84 & & & & & 1.57 & 1.33 & 1.93 & 1.24 \\
\hline $15 \mathrm{H}-2,95-100$ & 130.17 & CP16 & AP 13 & 34.02 & 2.13 & 1.27 & & & & & 1.73 & 1.62 & 2.05 & 1.09 \\
\hline $15 \mathrm{H}-3,95-100$ & 131.67 & CP16 & AP13 & 34.25 & 2.15 & 0.99 & & & & & 1.43 & & 1.94 & 1.09 \\
\hline $15 \mathrm{H}-4,95-100$ & 133.17 & CP16 & AP 13 & 34.47 & 2.06 & 1.07 & & & & & & & 2.00 & 1.04 \\
\hline $15 H-5,95-100$ & 134.67 & CP16 & AP13 & 34.70 & 2.26 & 1.17 & & & & & 1.34 & 1.71 & 2.15 & 1.00 \\
\hline $15 \mathrm{H}-6,95-100$ & 136.17 & CP16 & AP13 & 34.86 & 2.18 & 1.12 & & & & & 1.39 & 1.54 & 2.08 & 0.98 \\
\hline $15 \mathrm{H}-\mathrm{CC}$ & 137.53 & CP16 & AP13 & 35.01 & 2.17 & 1.34 & & & & & 1.23 & 1.62 & 2.17 & 1.01 \\
\hline $16 \mathrm{H}-1,89-94$ & 138.11 & CP16 & AP13 & 35.07 & 2.22 & 1.30 & & & & & 1.56 & 1.82 & 1.93 & 1.17 \\
\hline $16 \mathrm{H}-2,95-100$ & 139.67 & CP16 & AP13 & 35.24 & 2.52 & 1.49 & & & & & 1.55 & 1.73 & 2.31 & 1.25 \\
\hline $16 \mathrm{H}-3,95-100$ & 141.17 & CP16 & AP13 & 35.41 & 2.10 & 1.26 & 2.47 & 0.34 & & & 1.56 & 0.05 & 2.28 & 1.15 \\
\hline $16 \mathrm{H}-4,95-100$ & 142.67 & CP16 & AP13 & 35.57 & 1.71 & 0.98 & 2.50 & 0.04 & & & 1.73 & 2.00 & 2.02 & 1.22 \\
\hline $16 \mathrm{H}-5,95-100$ & 144.17 & CP16 & AP13 & 35.73 & 2.21 & 1.49 & 2.16 & -0.13 & & & 2.02 & 2.02 & 2.42 & 1.28 \\
\hline $16 \mathrm{H}-6,95-100$ & 145.67 & CP16 & AP13 & 35.90 & 2.16 & 1.43 & 2.91 & 0.45 & & & & & 2.08 & 1.32 \\
\hline $16 \mathrm{H}-\mathrm{CC}$ & 146.70 & CP16 & AP13 & 36.04 & 1.59 & 1.29 & 2.16 & -0.13 & & & & & & \\
\hline $17 H-1,28-33$ & 147.01 & CP16 & AP13 & 36.08 & 1.46 & 1.32 & 1.90 & & & & 1.03 & 1.97 & 1.60 & 0.90 \\
\hline $17 \mathrm{H}-\mathrm{CC}$ & 147.53 & CP16 & AP13 & 36.15 & 1.53 & 1.30 & 1.93 & 0.32 & & & 0.99 & 1.93 & 1.54 & 1.07 \\
\hline $18 \mathrm{H}-1,95-100$ & 148.57 & CP16 & AP13 & 36.30 & 1.44 & 0.54 & 1.80 & -0.17 & & & & & 1.45 & 1.15 \\
\hline $18 \mathrm{H}-2,95-100$ & 150.07 & CP16 & AP13 & 36.51 & 1.06 & 0.81 & & & & & 0.88 & 1.75 & 1.27 & 1.09 \\
\hline $18 \mathrm{H}-3,95-100$ & 151.57 & CP16 & AP13 & 36.71 & 1.33 & 1.05 & 1.38 & 0.19 & & & & & 1.54 & 1.28 \\
\hline $18 H-4,95-100$ & 153.07 & CP16 & AP13 & 36.92 & 1.14 & 1.04 & 1.50 & 0.04 & & & & & 1.42 & 1.30 \\
\hline $18 \mathrm{H}-5,95-100$ & 154.57 & CP16 & AP13 & 37.12 & 0.85 & 0.89 & 1.48 & 0.15 & & & 0.58 & 1.72 & 1.45 & 1.08 \\
\hline $18 H-6,95-100$ & 156.07 & CP16 & AP13 & 37.33 & 1.22 & 0.96 & 1.62 & -0.02 & & & 0.91 & 1.86 & 1.43 & 1.27 \\
\hline $18 \mathrm{H}-\mathrm{CC}$ & 157.10 & CP16 & AP13 & 37.48 & 1.80 & 1.00 & 1.73 & 0.32 & & & 1.12 & 1.84 & 1.45 & 1.30 \\
\hline $19 H-1,90-95$ & 158.07 & CP16 & AP12 & 37.61 & 1.27 & 1.24 & 1.45 & 0.09 & & & 0.91 & 1.86 & 1.13 & 1.13 \\
\hline $19 \mathrm{H}-2,90-95$ & 159.57 & CP16 & AP12 & 37.82 & 1.33 & 1.23 & 1.37 & 0.10 & & & 0.83 & 1.89 & 1.60 & 1.25 \\
\hline $19 \mathrm{H}-3,90-95$ & 161.07 & CP16 & AP12 & 38.02 & 1.01 & 1.31 & 1.50 & 0.35 & & & 1.01 & 2.15 & 1.47 & 1.44 \\
\hline $19 \mathrm{H}-4,90-95$ & 162.57 & CP15b & AP12 & 38.18 & 0.81 & 1.10 & 1.20 & 0.21 & & & 0.72 & 2.10 & 1.03 & 1.44 \\
\hline $19 H-5,90-95$ & 164.07 & CP15b & AP12 & 38.31 & 0.84 & 1.34 & 1.38 & 0.26 & & & 1.13 & 1.09 & 1.36 & 1.42 \\
\hline $19 H-6,90-95$ & 165.57 & CP15b & AP12 & 38.43 & 0.95 & 1.28 & 1.37 & 0.36 & & & 0.54 & 1.96 & 1.26 & 1.30 \\
\hline $19 \mathrm{H}-\mathrm{CC}$ & 166.07 & CP15b & AP12 & 38.48 & 1.18 & 1.15 & & & & & 0.36 & 1.93 & & \\
\hline $20 \mathrm{H}-1,95-100$ & 167.57 & CP15b & AP12 & 38.60 & 0.93 & 1.38 & & & & & 0.52 & 1.98 & & \\
\hline $20 \mathrm{H}-2,95-100$ & 169.07 & CP15b & AP12 & 38.73 & 0.71 & 0.90 & 1.11 & -0.07 & & & 0.38 & 1.84 & 1.08 & 0.97 \\
\hline $20 \mathrm{H}-3,95-100$ & 170.57 & CP15A & AP12 & 38.88 & 0.90 & 1.38 & 1.27 & 0.41 & & & 0.38 & 2.03 & 1.17 & 1.37 \\
\hline $20 \mathrm{H}-4,95-100$ & 172.07 & CP15A & AP12 & 39.12 & 1.29 & 1.29 & 1.46 & 0.57 & & & 0.54 & 2.22 & 1.27 & 1.57 \\
\hline $20 \mathrm{H}-5,95-100$ & 173.57 & CP15A & AP12 & 39.36 & 1.21 & 1.44 & 1.54 & 0.68 & & & 0.54 & 2.22 & 1.10 & 1.43 \\
\hline $20 \mathrm{H}-6,95-100$ & 175.07 & CP15A & AP12 & 39.59 & 1.28 & 1.22 & 1.40 & 0.39 & & & 0.32 & 1.92 & 1.22 & 1.23 \\
\hline
\end{tabular}


Table 3. Oxygen and carbon isotope ratios of foraminifer taxa from Hole 738B.

\begin{tabular}{|c|c|c|c|c|c|c|c|c|c|c|c|c|c|c|c|c|c|c|c|c|c|c|}
\hline \multirow{3}{*}{$\begin{array}{l}\text { Core, section, } \\
\text { interval (cm) }\end{array}$} & \multirow{4}{*}{$\begin{array}{l}\text { Depth } \\
\text { (ntest) }\end{array}$} & \multirow{3}{*}{$\begin{array}{l}\text { Marmo. } \\
\text { Biozone }\end{array}$} & \multirow{4}{*}{$\begin{array}{l}\text { Ant. formm. } \\
\text { Biozone }\end{array}$} & \multirow{4}{*}{$\begin{array}{c}\text { Age } \\
(m, y,) \\
\cdots\end{array}$} & \multicolumn{18}{|c|}{ SELECTED FORAMINIFER TAXA } \\
\hline & & & & & \multicolumn{2}{|c|}{ cibicidoides spp. } & \multicolumn{2}{|c|}{$\frac{\text { Stilostanellas }}{\text { subspinosa }}$} & \multicolumn{2}{|c|}{$\frac{\text { Nuttallides }}{\text { truempri }}$} & \multicolumn{2}{|c|}{$\frac{\text { chiloguembel ine }}{\text { spp. }}$} & \multicolumn{2}{|c|}{$\frac{\text { Acorinine }}{\text { primitive }}$} & \multicolumn{2}{|c|}{$\frac{\text { Globorotaloides: }}{\text { suteri }}$} & \multicolumn{2}{|c|}{$\begin{array}{l}\text { subbotine } \\
\text { engiporolides }\end{array}$} & \multicolumn{2}{|c|}{$\frac{\text { Subbotine }}{\text { linsperts }}$} & \multicolumn{2}{|c|}{$\frac{\text { Globiger inatheka }}{\text { index }}$} \\
\hline & & & & & 8180 & $813 \mathrm{c}$ & 8180 & $813 c$ & 8180 & $813 c$ & 5180 & $813 c$ & ô 180 & $813 \mathrm{c}$ & 8.180 & $\delta 13 c$ & $\delta 180$ & $\delta 13 c$ & 8180 & $\delta 13 c$ & 8180 & $\delta 13 c$ \\
\hline \multicolumn{20}{|l|}{$119-7388$} & & & \\
\hline $3 H-4,90-95$ & 18.42 & CP15b/CP16 & AP13 & L. olis. & 2.27 & 1.45 & & & & & & & & & & & & & & & & \\
\hline $3 \mathrm{H}-5,90-95$ & 20.42 & CP15b/CP16 & AP13 & t. otig. & 1.40 & 1.28 & & & & & & & & & & & & & & & & \\
\hline $3 \mathrm{H}-\mathrm{CC}$ & 23.00 & CP15b/CP16 & $A P 13$ & L. olig. & $1 . \pi$ & 1.36 & 1.95 & 0.15 & & & & & & & 1.91 & 1.51 & 1.61 & 1.82 & 1.22 & 1.67 & & \\
\hline $4 \mathrm{H}-1,90-95$ & 23.92 & CP15b/CP16 & AP13 & 36.00000 & 1.31 & 0.87 & & & & & 1.00 & 2.32 & & & 1.56 & 1.64 & & & & & & \\
\hline $6 H \cdot 1,90-95$ & 23.92 & CP15b/CP16 & AP13 & 36.00000 & 1.46 & 1.19 & & & & & & & & & 1.60 & 1.40 & & & & & & \\
\hline $4 \mathrm{H}-2,90-95$ & 25.42 & CP $15 \mathrm{~b} / \mathrm{CP} 16$ & AP13 & 36.26109 & 1.28 & 0.84 & 1.47 & -0.15 & & & 0.78 & 1.91 & & & 1.40 & 0.96 & & & & & & \\
\hline $6 \mathrm{H}-3,90-95$ & 26.92 & CP15b/CP16 & AP13 & 36.52219 & 0.93 & 0.97 & & & & & & & & & & & & & & & & \\
\hline $4 H-3,90-95$ & 26.92 & CP15b/CP16 & AP13 & 36.52219 & 1.06 & 0.99 & 1.26 & 0.12 & & & 0.83 & 2.01 & & & 1.31 & 1.36 & & & & & & \\
\hline $4 H \cdot 4,90-95$ & 28.62 & CP $15 \mathrm{~b} / \mathrm{CP} 16$ & $A P 13$ & 36.78328 & 1.21 & 0.91 & & & & & & & & & 1.38 & 1.27 & & & & & & \\
\hline $6 \mathrm{H}-4,90-95$ & 28.42 & $\mathrm{CP} 15 \mathrm{~b} / \mathrm{CP} 16$ & AP13 & 36.78328 & 0.92 & 0.80 & 1.48 & 0.16 & & & 1.10 & 2.09 & & & 1.50 & 1.54 & & & & & & \\
\hline $4 H-5,90-95$ & 29.92 & $\mathrm{CP} 15 \mathrm{~b} / \mathrm{CP} 16$ & AP13 & 37.04438 & 1.10 & 0.99 & & & & & & & & & 1.32 & 1.25 & & & & & & \\
\hline $4 H-5,90-95$ & 29.92 & CP15b/CP16 & AP13 & 37.04638 & 0.82 & 0.85 & & & & & & & & & 1.25 & 1.45 & & & & & & \\
\hline $4 H-6,90-95$ & 31.42 & CP15b/CP16 & $A P 13$ & 37.30548 & 1.09 & 0.72 & 1.37 & 0.05 & & & 1.05 & 1.96 & & & 1.40 & 1.17 & & & & & & \\
\hline $6 \mathrm{H}-7,90-95$ & 32.86 & CP15b/CP16 & AP13 & 37.55265 & 1.30 & 0.99 & 2.15 & 0.44 & & & 1.19 & 1.99 & & & 1.49 & 1.23 & & & & & & \\
\hline $5 \mathrm{H}-1,90-95$ & 33.42 & CP15b/CP16 & AP13 & 37.65361 & 1.48 & 1.29 & 1.85 & 0.41 & & & & & & & 1.32 & 1.37 & 1.51 & 1.72 & & & & \\
\hline $5 \mathrm{H}-2,90-95$ & 36.92 & CP15b/CP16 & AP13 & 37.91470 & 1.19 & 1.41 & & & & & & & & & 1.31 & 1.30 & & & & & & \\
\hline $5 \mathrm{H}-3,90-95$ & 36.42 & CP15b/CP16 & AP12 & 38.17565 & 1.22 & 1.30 & 1.58 & 0.13 & & & & & & & 1.53 & 1.25 & 1.46 & 1.52 & 1.17 & 1.52 & & \\
\hline $5 \mathrm{H}-4,90-95$ & 37.92 & CP15b/CP16 & AP12 & 38.43652 & 0.83 & 0.94 & & & & & & & & & 0.99 & 1.06 & & & & & & \\
\hline $5 H-5,90-95$ & 38.42 & CP15。 & AP12 & 38.69739 & 1.15 & 1.20 & & & & & & & & & 1.21 & 1.20 & 1.33 & 1.61 & & & & \\
\hline $5 H-6,90-95$ & 40.92 & CP15。 & AP12 & 38.86880 & 0.91 & 1.16 & & & & & & & & & 1.28 & 1.21 & & & & & & \\
\hline $5 \mathrm{H}-7,10-15$ & 41.12 & CP15. & AP12 & 38.88392 & 0.94 & 1.26 & & & & & & & & & 1.31 & 1.20 & & & & & & \\
\hline $5 \mathrm{H}-\mathrm{CC}$ & 62.00 & CP15, & AP12 & 38.95046 & 1.41 & 1.39 & 1.48 & 0.62 & & & 0.94 & 1.90 & & & 1.49 & 1.68 & 1.29 & 1.69 & 1.48 & 1.89 & & \\
\hline $6 H-1,90-95$ & 62.92 & CP15a & AP12 & 39.02001 & 1.19 & 1.18 & & & & & & & & & 1.46 & 1.16 & & & & & & \\
\hline $6 \mathrm{H}-2,90-95$ & 44.42 & CP15. & AP12 & 39.13343 & 1.26 & 0.79 & 1.38 & -0.12 & & & 0.51 & 1.92 & & & 1.25 & 0.78 & & & & & & \\
\hline $6 H-4,90-95$ & 47.42 & CP15. & AP12 & 39.36025 & 1.51 & 0.41 & & & & & & & & & 1.49 & 0.40 & & & & & & \\
\hline $6 \mathrm{H}-5,86-89$ & 48.92 & CP15。 & AP12 & 39.67366 & 1.16 & 0.85 & & & & & & & & & 1.35 & 0.72 & & & & & & \\
\hline $6 \mathrm{H}-6,84-89$ & 50.36 & CP15a & AP12 & 39.58254 & 1.34 & 0.88 & & & & & & & & & 1.35 & 0.69 & & & & & & \\
\hline $6 \mathrm{H}-\mathrm{CC}$ & 51.50 & CP15, & XP12 & 39.68873 & 1.02 & 0.68 & 0.51 & 0.45 & & & & & & & 2.16 & 1.03 & & & 1.68 & 1.16 & 1.00 & 2.03 \\
\hline $7 \mathrm{H}-\mathrm{CC}$ & 61.00 & CP15a & AP12 & 40.38701 & 1.03 & 1.05 & 1.52 & 0.01 & 0.96 & 0.84 & & & & & 1.57 & 1.06 & & & 1.59 & 1.50 & 1.53 & 1.87 \\
\hline $\mathrm{BH}-\mathrm{CC}$ & 70.50 & CP15, & AP11 & 41.40259 & 0.94 & 0.96 & 1.44 & -0.17 & & & & & & & & & & & 1.03 & 1.49 & 0.43 & 2.45 \\
\hline $9 \mathrm{H}-\mathrm{CC}$ & 80.00 & CP16日 & AP11 & 41.75670 & 0.73 & 1.06 & 1.12 & 0.00 & 0.67 & 0.62 & & & & & 1.13 & 1.20 & & & 0.95 & 1.60 & 0.47 & 2.46 \\
\hline $10 \mathrm{H}-\mathrm{CC}$ & 85.00 & CP16。 & AP10 & 41.94002 & & & & & & & & & 0.63 & 2.51 & 1.13 & 1.47 & & & 0.91 & 1.44 & & \\
\hline $11 \mathrm{H}-\mathrm{CC}$ & 95.37 & CP160 & AP10 & 42.32438 & & & & & & & & & -0.23 & 2.14 & & & & & & & 0.02 & 2.46 \\
\hline
\end{tabular}




\begin{tabular}{|c|c|c|c|c|c|c|c|c|c|c|c|c|c|c|c|c|c|c|c|c|c|c|}
\hline \multirow{3}{*}{$\begin{array}{l}\text { Core, section, } \\
\text { intervat (cm) }\end{array}$} & \multirow{3}{*}{$\begin{array}{l}\text { Depth } \\
\text { (nost) }\end{array}$} & \multirow{3}{*}{$\begin{array}{l}\text { Marno. } \\
\text { Blozone }\end{array}$} & \multirow{3}{*}{$\begin{array}{l}\text { Ant. fornam. } \\
\text { Biozone }\end{array}$} & \multirow{3}{*}{$\begin{array}{c}\text { Age } \\
\left(m . y_{.}\right) \\
\cdots . . . .\end{array}$} & \multicolumn{18}{|c|}{ SELECTED FORNMINIFER TAXA } \\
\hline & & & & & \multicolumn{2}{|c|}{ ciblcidoides spp. } & \multicolumn{2}{|c|}{$\frac{\text { Stllostonello }}{\text { subspinoso }}$} & \multicolumn{2}{|c|}{$\frac{\text { Nuttall ides }}{\text { trueapy! }}$} & \multicolumn{2}{|c|}{$\frac{\text { chilloguenbel ine }}{\text { spp. }}$} & \multicolumn{2}{|c|}{$\frac{\text { Acarinino }}{\text { primitivo }}$} & \multicolumn{2}{|c|}{$\frac{\text { Globorotoloides: }}{\text { suteri }}$} & \multicolumn{2}{|c|}{ subootine } & \multicolumn{2}{|c|}{$\frac{\text { Subbotina }}{1 \text { inaperte }}$} & \multicolumn{2}{|c|}{$\frac{\text { Globiger inatheke }}{\text { index }}$} \\
\hline & & & & & 8180 & $\delta 13 c$ & 8180 & $513 c$ & 8180 & $813 c$ & $\delta 180$ & $\delta 13 c$ & $\delta 180$ & $\delta 13 c$ & 8180 & $813 \mathrm{C}$ & 8180 & $\delta 13 c$ & $\delta 180$ & $813 \mathrm{C}$ & 8180 & $813 \mathrm{c}$ \\
\hline $12 \mathrm{~K}-1,87-92$ & 98.39 & CP16e & Ap10 & 42.21238 & -0.01 & 1.13 & & & 0.20 & 0.56 & & & 0.15 & 2.36 & & & & & & & 0.06 & 2.36 \\
\hline $12 \mathrm{~K}-3,87-92$ & 101.39 & CP160 & AP10 & 42.55642 & 0.05 & 0.88 & & & & & & & 0.05 & 2.04 & & & & & & & 0.12 & 2.01 \\
\hline $12 \mathrm{H}-5,87-92$ & 106.02 & CP148 & AP10 & 42.85802 & 0.53 & 0.93 & & & 0.26 & 0.56 & & & 0.07 & 2.26 & & & & & & & 0.31 & 2.53 \\
\hline $15 x-c c$ & 127.79 & CP13 & AP10 & 45.17559 & 0.43 & 0.80 & & & & & & & 0.17 & 2.02 & & & & & & & 0.01 & 2.21 \\
\hline $17 x-c c$ & 166.80 & CP13 & APQ & 46.31166 & 0.35 & 0.93 & & & 0.20 & 0.49 & & & 0.22 & 0.49 & & & & & & & & \\
\hline $18 x-2,88-93$ & 169.20 & CP13 & APB & 46.38332 & & & & & 0.31 & 0.46 & & & 0.28 & 1.04 & & & & & 0.46 . & 1.12 & & \\
\hline $18 x-c c$ & 156.50 & CP13 & APB & 46.60128 & & & & & 0.08 & -0.03 & & & & & & & & & & & & \\
\hline $19 x-2,90-95$ & 158.92 & CP13 & APB & 46.67356 & & & & & 0.28 & 0.18 & & & 0.23 & 2.26 & & & & & & & & \\
\hline $19 x-4,90-95$ & 161.92 & CP13 & APB & 46.76311 & 0.52 & 0.74 & & & & & & & 0.07 & 2.11 & & & & & 0.44 & 1.27 & & \\
\hline $19 x-6, c c$ & 164.92 & CP13 & APB & 46.85268 & & & & & & & & & 0.12 & 1.57 & & & & & & & & \\
\hline $19 x-c c$ & 166.20 & CP13 & APB & 46.89090 & & & & & 0.39 & 0.32 & & & 0.22 & 1.73 & & & & & & & & \\
\hline $20 x-2,90-95$ & 168.62 & CP13 & APB & 46.96316 & & & & & 0.14 & 0.08 & & & -0.40 & 2.26 & & & & & & & & \\
\hline $20 x-4,90-95$ & 171.62 & CP13 & APB & 47.05273 & & & & & 0.28 & 0.26 & & & 0.03 & 1.71 & & & & & 0.49 & 1.10 & & \\
\hline $20 x-6,90-95$ & 174.62 & CP13 & APB & 47.14230 & & & & & -0.19 & -0.07 & & & -0.17 & 2.18 & & & & & 0.39 & 1.05 & & \\
\hline $20 x-c c$ & 175.80 & CP13 & APB & 47.17753 & & & & & & & & & -0.10 & 1.86 & & & & & & & & \\
\hline $21 x-1,90-95$ & 176.72 & CP13 & APB & 47.20500 & & & & & 0.09 & 0.34 & & & & & & & & & & & & \\
\hline $21 x-3,90-95$ & 179.72 & CP13 & APB & 47.29457 & & & & & 0.06 & 0.14 & & & -0.07 & 2.08 & & & & & & & & \\
\hline $21 x-c c$ & 185.40 & CP13 & APB & 47.46417 & 0.33 & 0.57 & & & 0.17 & 0.25 & & & -0.31 & 1.97 & & & & & 0.27 & 1.05 & & \\
\hline $22 x-2,90-95$ & 187.82 & CP13 & AP7 & 47.69445 & 0.16 & 0.29 & & & -0.06 & -0.01 & & & -0.23 & 2.01 & & & & & 0.23 & 0.90 & & \\
\hline $22 x-4,90-95$ & 190.82 & CP13 & AP7 & 48.17262 & & & & & & & & & -0.90 & 2.02 & & & & & 0.13 & 1.29 & & \\
\hline $22 x-c c$ & 195.00 & CP13 & AP7 & 48.83887 & & & & & -0.14 & 0.10 & & & -0.47 & 2.12 & & & & & 0.02 & 1.05 & & \\
\hline $23 x-1,90-95$ & 195.92 & CP13 & AP7 & 48.98551 & & & & & & & & & -0.01 & 1.75 & & & & & 0.00 & 1.10 & & \\
\hline $23 x-c c$ & 204.60 & CP13 & AP7 & 50.18460 & & & & & & & & & -0.56 & 1.53 & & & & & & & & \\
\hline $24 x-2,90-95$ & 207.02 & CP13 & AP7 & 50.44532 & -0.37 & 0.86 & & & -0.16 & 0.69 & & & -0.97 & 2.88 & & & & & 0.13 & 1.29 & & \\
\hline $24 x-c c$ & 214.30 & CP13 & AP7 & 51.22962 & -0.34 & 0.92 & & & & & & & & & & & & & 0.03 & 1.38 & & \\
\hline
\end{tabular}


Table 4. Oxygen and carbon isotope ratios of foraminifer taxa from Hole $738 \mathrm{C}$.

\begin{tabular}{|c|c|c|c|c|c|c|c|c|c|c|c|c|c|c|c|c|c|c|c|c|}
\hline \multirow{3}{*}{$\begin{array}{l}\text { Core, section, } \\
\text { interval (cm) }\end{array}$} & \multirow{3}{*}{$\begin{array}{l}\text { Depth } \\
\text { (mbsf) }\end{array}$} & \multirow[t]{3}{*}{$\begin{array}{l}\text { Namo. } \\
\text { biozone }\end{array}$} & \multirow{3}{*}{$\begin{array}{l}\text { Ant. foram } \\
\text { biozone }\end{array}$} & \multirow{3}{*}{$\begin{array}{c}\text { Age } \\
(m . y .)\end{array}$} & & \multicolumn{15}{|c|}{ SELECTED FORAMINIFER IAXA } \\
\hline & & & & & \multicolumn{2}{|c|}{ cibicidoides spp. } & \multicolumn{2}{|c|}{$\frac{\text { Nuttallides }}{\text { truempyi }}$} & \multicolumn{2}{|c|}{$\begin{array}{l}\text { Gavel inella } \\
\text { beccariiformis }\end{array}$} & \multicolumn{2}{|c|}{$\frac{\text { Chiloguenbel ina }}{\text { spp. }}$} & \multicolumn{2}{|c|}{$\frac{\text { Subbotina }}{\text { linaperta }}$} & \multicolumn{2}{|c|}{$\frac{\text { Acarinina }}{\text { erimitive }}$} & \multicolumn{2}{|c|}{$\frac{\text { subbotina }}{\text { spp. }}$} & \multicolumn{2}{|c|}{$\frac{\text { Acarinina }}{\text { spp. }}$} \\
\hline & & & & & 8180 & $\delta 13 c$ & 8180 & $813 \mathrm{C}$ & 8180 & $813 c$ & $\delta 180$ & $813 c$ & 8180 & $\delta 13 c$ & 8180 & $813 c$ & 8180 & $813 c$ & 8180 & $\delta 13 c$ \\
\hline \multicolumn{21}{|l|}{$119-738 \mathrm{C}$} \\
\hline $2 R-1,124-129$ & 197.86 & CP13 & $A P 7$ & 49.29 & -0.21 & 0.84 & 0.48 & 0.75 & & & & & & & & & & & & \\
\hline $2 R-C C$ & 206.20 & CP12 & AP7 & 50.36 & -0.41 & 0.84 & & & & & & & -0.06 & 1.36 & & & & & & \\
\hline $3 R-1,59-6$ & 206.79 & CP12 & AP7 & 50.42 & -0.52 & 1.23 & & & & & & & & & -0.58 & 2.23 & -0.11 & 1.38 & & \\
\hline $3 R-c c$ & 215.90 & CP12 & $A P 7$ & 51.40 & -0.50 & 1.20 & -0.63 & 0.70 & & & & & & & & & -0.21 & 1.42 & -0.65 & 2.14 \\
\hline $4 R-1,90-95$ & 216.82 & $C P 12$ & AP7 & 51.50 & -0.52 & 1.13 & -0.67 & 0.81 & & & & & & & & & -0.16 & 1.52 & -0.77 & 2.54 \\
\hline $4 R-3,90-95$ & 219.82 & CP12 & AP7 & 51.82 & & & -0.66 & 0.74 & & & & & & & -0.33 & 1.80 & & & & \\
\hline $4 R-C C$ & 225.60 & CP12 & AP7 & 52.45 & & & -0.67 & 0.74 & & & & & & & -0.87 & 2.08 & -0.37 & 1.27 & & \\
\hline $5 R-2,73-78$ & $227.85 \mathrm{C}$ & CP10/CP11 & AP7 & 52.64 & & & -0.67 & 1.02 & & & & & & & & & -1.12 & 1.21 & -0.43 & 1.97 \\
\hline $5 R-C C$ & $235.20 \mathrm{C}$ & CP10/CP11 & AP7 & 52.95 & & & -0.88 & 0.89 & & & & & & & -0.57 & 1.99 & -0.28 & 1.65 & & \\
\hline $6 R-1,84-89$ & $236.06 \mathrm{C}$ & CP10/CP11 & AP7 & 52.99 & & & & & & & & & & & -0.86 & 2.04 & -0.37 & 1.29 & & \\
\hline $6 R-C C$ & $244.80 \mathrm{C}$ & CP10/CP11 & $A P 7$ & 53.36 & & & -0.82 & 0.83 & & & & & & & & & -0.53 & 1.37 & & \\
\hline $7 R-1,88-93$ & $245.66 \mathrm{c}$ & CP10/CP11 & AP7 & 53.40 & -0.75 & 0.79 & -0.65 & 0.81 & & & & & & & & & -0.57 & 0.94 & -0.76 & 2.14 \\
\hline $7 R-3,23 \cdot 28$ & $247.83 \mathrm{C}$ & CP10/CP11 & APGb & 53.49 & & & & & & & & & & & & & -0.31 & 1.22 & -0.69 & 1.39 \\
\hline $7 R-C C$ & $254.40 \mathrm{C}$ & CP10/CP11 & APGb & $53 . \pi 7$ & -0.84 & 0.55 & -0.72 & 0.26 & & & & & & & & & -0.47 & 0.97 & -0.86 & 1.60 \\
\hline $7 R-C C$ & $254.40 \mathrm{C}$ & CP10/CP11 & APSb & 53.77 & & & -0.90 & 0.13 & & & & & & & & & & & & \\
\hline $8 R-1,90-95$ & $255.32 \mathrm{C}$ & CP10/CP11 & APGb & 53.81 & & & -0.96 & 0.63 & & & & & & & & & -0.46 & 1.15 & -1.11 & 1.91 \\
\hline$B R \cdot C C$ & 264.10 & CPQ & APSa & 54.47 & & & -0.78 & 0.62 & & & & & & & & & 0.11 & 0.86 & & \\
\hline $98-1,25-30$ & 264.36 & CP9 & APGa & 54.50 & -0.48 & 0.51 & -0.77 & 0.03 & & & & & & & & & -0.60 & 0.72 & -1.36 & 1.87 \\
\hline $9 R-\mathrm{CC}$ & 273.80 & CP9 & APGa & 55.51 & -0.62 & 0.35 & -0.58 & 0.22 & & & & & & & & & -0.44 & 0.71 & & \\
\hline $9 R-C C$ & 273.80 & $C P 9$ & APGa & 55.51 & & & -0.65 & 0.09 & & & & & & & & & & & & \\
\hline $10 R-1,95-100$ & 274.77 & CP9 & APGa & 55.61 & & & -0.98 & -0.72 & & & & & & & & & & & -0.60 & 3.08 \\
\hline $10 R-1,95-100$ & 274.77 & CP9 & APGo & 55.61 & -0.72 & -0.18 & -0.72 & -0.30 & & & -0.60 & 0.08 & & & & & -0.65 & 0.62 & -1.18 & 1.62 \\
\hline $10 R-2,95-100$ & 276.25 & $C P 9$ & APBO & 55.77 & & & & & & & & & & & & & & & -1.42 & 2.30 \\
\hline $10 R-3,98-102$ & 277.80 & CP9 & APGa & 55.94 & -0.47 & 0.12 & -0.59 & -0.19 & & & -0.36 & 0.22 & & & & & -0.82 & 1.15 & & \\
\hline $10 R-3,98-102$ & 277.80 & CP9 & APGa & 55.94 & & & -0.45 & 0.03 & & & & & & & & & & & & \\
\hline $10 R-C c$ & 283.40 & CP9 & APBO & 56.54 & -0.25 & 0.32 & -0.43 & 0.06 & & & -0.22 & 0.41 & & & & & -0.34 & 0.95 & -1.05 & 1.96 \\
\hline $11 R-1,115-118$ & 284.52 & CPB & APS & 57.80 & -0.14 & 0.99 & -0.12 & 0.40 & & & & & & & & & -0.35 & 1.26 & -0.73 & 2.31 \\
\hline $11 R-2,115-118$ & 286.02 & CP8 & APS & 57.87 & & & & & -0.30 & 0.81 & & & & & & & -0.13 & 1.58 & -0.73 & 2.90 \\
\hline $11 R-c C$ & 293.00 & CPB & APS & 58.22 & 0.08 & 1.26 & -0.18 & 0.90 & 0.01 & 0.04 & & & & & & & 0.05 & 1.65 & -0.45 & 2.63 \\
\hline $13 R-C C$ & 312.30 & CP6/CP7 & APG & 59.20 & & & -0.10 & 1.95 & -0.29 & 1.88 & & & & & & & -0.08 & 2.99 & & \\
\hline $13 R-C C$ & 312.30 & $C P 6 / C P 7$ & AP4 & 59.20 & & & 0.03 & 1.90 & 0.57 & 2.48 & & & & & & & & & -0.61 & 3.73 \\
\hline $14 R \cdot C C$ & 321.90 & CP $6 / C P 7$ & AP4 & 59.76 & & & 0.02 & 2.43 & 0.02 & 2.38 & & & & & & & & & & \\
\hline $14 R-C C$ & 321.90 & $\mathrm{CP} 6 / \mathrm{CP} 7$ & AP4 & 59.76 & & & 0.07 & 2.16 & & & & & & & & & 0.05 & 3.05 & -0.58 & 4.23 \\
\hline
\end{tabular}




\begin{tabular}{|c|c|c|c|c|c|c|c|c|c|c|c|c|c|c|c|c|c|c|c|c|}
\hline \multirow{3}{*}{$\begin{array}{l}\text { Core, section, } \\
\text { interval }(\mathrm{cm})\end{array}$} & \multirow{3}{*}{$\begin{array}{l}\text { Depth } \\
\text { (nibsf) }\end{array}$} & \multirow{3}{*}{$\begin{array}{l}\text { Nomo. } \\
\text { biozone }\end{array}$} & \multirow{3}{*}{$\begin{array}{l}\text { Ant. foram } \\
\text { biozone }\end{array}$} & \multirow{3}{*}{$\begin{array}{l}\text { Age } \\
\text { (m.y.) }\end{array}$} & \multicolumn{16}{|c|}{ SELECIED FORAMIMIFER TAXA } \\
\hline & & & & & Cibicidoid & s spp. & \multicolumn{2}{|c|}{$\frac{\text { Nut tallides }}{\text { truempyi }}$} & \multicolumn{2}{|c|}{ Gecelinello } & \multicolumn{2}{|c|}{$\frac{\text { Chiloguenbel ina }}{\text { spp. }}$} & \multicolumn{2}{|c|}{ Subbotina } & \multicolumn{2}{|c|}{$\frac{\text { Acarinins }}{\text { primitivo }}$} & \multicolumn{2}{|c|}{$\frac{\text { Subbotina }}{\text { spp. }}$} & \multicolumn{2}{|c|}{$\frac{\text { Acarinina }}{\text { spp. }}$} \\
\hline & & & & & 8180 & $813 \mathrm{C}$ & 8180 & $513 \mathrm{c}$ & \& 180 & $\delta 13 \mathrm{C}$ & 8180 & o $13 \mathrm{C}$ & 8180 & $813 \mathrm{C}$ & $\delta 180$ & $\delta 13 c$ & 8180 & $\delta 13 c$ & 5180 & $813 c$ \\
\hline $15 R-1,90-95$ & 322.82 & CP6/CP7 & AP4 & 60.24 & & & & & & & & & & & & & & & -0.49 & 3.53 \\
\hline $15 R \cdot C C$ & 331.60 & CPS & $\mathrm{AP4}$ & 61.33 & & & 0.50 & 1.64 & & & & & & & & & & & -0.42 & 3.57 \\
\hline $15 R-C C$ & 331.60 & CPS & $A P 4$ & 61.33 & & & 0.42 & 1.66 & 0.50 & 1.41 & & & & & & & & & & \\
\hline $16 R-1,55-60$ & 332.17 & CP5 & $A P 4$ & 61.40 & & & -0.55 & 0.91 & & & & & & & & & 0.26 & 1.95 & & \\
\hline $16 R \cdot 2,74-76$ & 333.86 & CP5 & $A P 4$ & 61.70 & & & 0.13 & 0.99 & -0.29 & 1.88 & & & & & & & & & -0.54 & 2.75 \\
\hline $16 R \cdot 2,74 \cdot 76$ & 333.86 & CP5 & $A P 4$ & 61.70 & 0.16 & 1.14 & 0.22 & 1.08 & -0.11 & 0.57 & & & & & & & 0.03 & 1.81 & & \\
\hline $16 R-4,90-95$ & 337.02 & CP4 & $A P 3$ & 62.27 & & & 0.11 & 0.68 & 0.19 & 0.68 & & & & & & & & & -0.65 & 2.52 \\
\hline $16 R \cdot 4,90-95$ & 337.02 & CP4 & $A P 3$ & 62.27 & & & 0.00 & 0.91 & 0.22 & 0.74 & & & & & & & -0.01 & 1.62 & & \\
\hline $16 R-5,90-93$ & 338.52 & CP4 & AP2 & 62.44 & & & -0.03 & 0.98 & 0.13 & 0.79 & & & & & & & & & & \\
\hline $16 R-6,90-93$ & 340.02 & $C P 6$ & AP2 & 62.53 & & & & & 0.22 & 0.57 & & & & & & & & & & \\
\hline $16 R-C C$ & 361.30 & CP6 & $A P 2$ & 62.61 & & & 0.19 & 1.10 & 0.19 & 0.86 & & & & & & & & & & \\
\hline $17 R-1,90-95$ & 342.22 & CP4 & AP2 & 62.66 & & & 0.03 & 0.71 & -0.10 & 0.62 & -0.31 & 1.67 & & & & & 0.17 & 1.40 & & \\
\hline $17 R-2,90-95$ & 343.72 & CP4 & AP2 & 62.69 & & & 0.06 & 0.85 & -0.08 & 0.64 & & & & & & & & & & \\
\hline $17 R-3,90-95$ & 345.22 & CP4 & AP2 & 62.77 & & & 0.12 & 0.91 & 0.16 & 0.84 & -0.28 & 1.88 & & & & & & & & \\
\hline $17 R-4,88-93$ & 346.72 & $\mathrm{CP} 4$ & $A P 2$ & 62.84 & & & -0.23 & 0.82 & & & & & & & & & -0.13 & 1.26 & & \\
\hline $17 R-5,90-95$ & 348.22 & CP4 & AP2 & 63.00 & & & 0.19 & 0.98 & 0.02 & 0.72 & -0.43 & 1.72 & & & & & 0.16 & 1.31 & & \\
\hline $17 R-7,65-70$ & 350.47 & CP4 & $A P 2$ & 63.14 & & & 0.12 & 0.80 & 0.02 & 0.78 & & & & & & & 0.21 & 1.26 & & \\
\hline $17 R-C C$ & 350.90 & $\mathrm{CP} 4$ & $A P 1 b$ & 63.16 & & & & & 0.20 & 0.87 & & & & & & & 0.17 & 1.37 & & \\
\hline $18 R-1,94-96$ & 351.61 & $\mathrm{CP} 4$ & $A P 1 b$ & 63.20 & & & -0.21 & 0.76 & & & & & & & & & 0.25 & 1.56 & & \\
\hline $18 R-2,94-96$ & 353.11 & $\mathrm{CP} 4$ & AP1b & 63.26 & & & -0.20 & 0.77 & -0.06 & 0.82 & & & & & & & 0.16 & 1.42 & & \\
\hline $18 R \cdot C C$ & 360.50 & $\mathrm{CP} 2 / \mathrm{CP3}$ & $A P 1 b$ & 63.52 & & & 0.01 & 1.14 & -0.12 & 0.91 & & & & & & & 0.13 & 1.58 & & \\
\hline $19 R \cdot 2,88-90$ & 362.90 & $\mathrm{CPZ/CP3}$ & $A P 1 b$ & 63.85 & & & & & -0.26 & 1.09 & & & & & & & & & & \\
\hline $19 R-3,88-90$ & 364.90 & $\mathrm{CP2} / \mathrm{CP} 3$ & AP1b & 64.17 & & & & & -0.49 & 0.84 & & & & & & & & & & \\
\hline $198-C C$ & 370.20 & cP1b & $A P 1 b$ & 65.04 & & & -0.16 & 1.61 & & & & & & & & & & & & \\
\hline $20 R-1,102-104$ & 371.23 & cP1b & AP 1b & 65.21 & & & -0.32 & 1.40 & -0.46 & 1.25 & & & & & & & & & & \\
\hline $20 R-2,118-120$ & 372.90 & CP1b & AP1b & 65.49 & & & -0.31 & 1.44 & -0.26 & 1.31 & & & & & & & & & & \\
\hline $20 R-3,90-92$ & 374.12 & CP1b & AP1B & 65.69 & & & -0.40 & 1.56 & -0.20 & 1.62 & & & & & & & & & & \\
\hline $20 R \cdot 5,34-35$ & 377.23 & CP1b & AP18 & 66.19 & & & -0.61 & 2.13 & -0.45 & 1.33 & -0.52 & 1.08 & & & & & & & & \\
\hline $20 R-5,34-35$ & 377.23 & $\mathrm{CP} 1 \mathrm{~b}$ & AP1a & 66.19 & & & & & & & & & & & & & & & & \\
\hline $20 R-5,90-92$ & 377.80 & CP10 & AP & 66.27 & & & & & -0.20 & 1.62 & & & & & & & & & & \\
\hline $21 R-C C$ & 380.70 & NC23 & A. may. & 66.54 & & & -0.49 & 1.50 & & & & & & & & & & & & \\
\hline
\end{tabular}


Table 5. Mean values of intertaxa isotopic differences.

\begin{tabular}{lcccc}
\hline & \multicolumn{2}{c}{ Cibicidoides spp. } & \multicolumn{2}{c}{ Gavelinella beccariformis } \\
& $\delta^{18} \mathrm{O}$ & $\delta^{13} \mathrm{C}$ & $\delta{ }^{18} \mathrm{O}$ & $\delta^{13} \mathrm{C}$ \\
\hline Nuttallides truempyi & $0.03 \pm 0.21$ & $0.28 \pm 0.15$ & $0.0 \pm 0.21$ & $-0.21 \pm 0.27$ \\
Stilostomella subspinosa & $\mathrm{N}=19$ & $\mathrm{~N}=19$ & $\mathrm{~N}=22$ & $\mathrm{~N}=22$ \\
& $-0.35 \pm 0.22$ & $0.88 \pm 0.24$ & & \\
& $\mathrm{~N}=55$ & $\mathrm{~N}=55$ & & \\
\hline
\end{tabular}

Note: Values are means of the difference between $\delta^{18} \mathrm{O}$ of the taxon in column and the taxon in row in the same sample. $\mathrm{N}=$ the number of samples used in the calculation.

Table 6. Adjustment for isotopic disequilibrium of benthic foraminifers.

\begin{tabular}{lcc}
\hline & $\delta^{18} \mathrm{O}$ & ${ }^{13} \mathrm{C}$ \\
\hline Cibicidoides spp. & 0.5 & 0.0 \\
Nuttallides truempyi & 0.5 & 0.0 \\
Gavelinella beccariiformis & 0.5 & 0.0 \\
Stilostomella subspinosa & 0.0 & 0.0 \\
\hline
\end{tabular}

Note: Adjustments made as noted in figure captions to compositions of benthic foraminifer taxa to correct for isotopic disequilibrium.

tion of the water column between intermediate and near-surface/surface water depths as reflected in the difference between $\delta^{18} \mathrm{O}$ values of benthic and planktonic foraminifers (Figs $3 \mathrm{~A}$ and 4). In contrast to the variable shallow-water foraminifer $\delta^{18} \mathrm{O}$ values which may reflect primarily seasonal temperature changes, the less variable values of benthic foraminifer and deeper-dwelling planktonic species reflect the relatively constant conditions of their habitat. Changes in the difference between $\delta^{18} \mathrm{O}$ values of benthic foraminifers and Subbotina spp. indicate that on the average the near-surface to bottom temperature difference was large in the Paleocene and earliest early Eocene (Figs. 3A and 4). It was small from 55 to about $51 \mathrm{Ma}$, and increased slightly after $51 \mathrm{Ma}$. In general, the surface to bottom difference also decreased from about 55.5 to $51 \mathrm{Ma}$ (Fig. 4).

The $\delta^{13} \mathrm{C}$ difference between surface or near-surface and intermediate waters reflects the removal of ${ }^{12} \mathrm{C}$ in the surface waters by productivity and the addition at depth of ${ }^{12} \mathrm{C}$ from the oxidation of organic matter. It can vary with the level of productivity in surface water or modifications in the properties of the deeper water. In the geologic record, the $\delta^{13} \mathrm{C}$ gradient can be inferred measuring the difference between the ${ }^{13} \mathrm{C} /{ }^{12} \mathrm{C}$ ratios of planktonic and benthic foraminifers. The inferred gradient can also be influenced but to a much smaller extent by other factors such as changes in the season or the depth of calcification of the planktonic foraminifer. At Site 738, the $\delta^{13} \mathrm{C}$ difference between near-surface to intermediate water was between 0.5 and $1 \% 0$ in the Paleocene, with the lower values in the older part of the record (Fig. 4). Near the maximum warming, this difference (Subbotina minus Nuttallides) decreased and preliminary $\delta^{18} \mathrm{O}$ and $\delta^{13} \mathrm{C}$ data of Chiloguembelina spp. from Site 738 suggest the almost complete elimination of isotopic gradients (Figs. 3A and 3B) similar to that reported for the Maud Rise Site 690 at about this time (Kennett and Stott, 1990). In the polar Indian Ocean in the early Eocene from about $55 \mathrm{Ma}$, the near-surface to bottom $\delta^{13} \mathrm{C}$ difference became more variable and values fluctuated closer to $0.5 \%$ before increasing to about $1 \%$ in the early middle Eocene (Fig. 4). The surface to bottom $\delta^{13} \mathrm{C}$ difference was low, close to about $1 \%$, from about 54 to 51 $\mathrm{Ma}$, relative to earlier values of about $2 \%$.

The changes in the $\delta^{13} \mathrm{C}$ and $\delta^{18} \mathrm{O}$ gradients can be correlated with changes in climate at this high-latitude location. In gen- eral, with the warming of the poles in the early Eocene, the gradients decreased, whereas they were higher during colder periods (Figs. 3 and 4). The decrease in foraminifer isotopic differences can be interpreted in terms of changes in the vertical thermal stratification of the water column. The decrease in the $\delta^{18} \mathrm{O}$ difference between the benthic and planktonic taxa to values close to zero indicates that the thermal stratification of the water column from the thermocline to intermediate-water depths was low or nonexistent during the early and earliest middle Eocene (Fig. 4). At this time the surface to bottom $\delta^{13} \mathrm{C}$ difference was also small. The isotopic difference between surface and near-surface waters did not significantly change during the $\mathrm{Pa}$ leocene/Eocene transition and in the earliest early Eocene. A slight decrease in this difference occurred during the late early Eocene after $54 \mathrm{Ma}$ with the increase of surface-water $\delta^{18} \mathrm{O}$. Hence, the reduction of isotopic differences between the surface and intermediate waters does not appear to have resulted from differential changes within the upper level of the water column because surface and near-surface waters varied sympathetically most of the time. The changes in the gradient could represent a decoupling of intermediate waters from the behavior of the upper layers of the water column.

The pattern of change in $\delta^{18} \mathrm{O}$ values of the foraminifer taxa suggests that intermediate waters may have formed at a different location than the polar regions in the early Eocene. We note that $\delta^{18} \mathrm{O}$ of the planktonic taxa had a minimum near $56 \mathrm{Ma}$ and increased gradually thereafter. Intermediate waters, as reflected by $\delta^{18} \mathrm{O}$ of $N$. truempyi were warm from about 56 to 54 $\mathrm{Ma}$ and began a gradual cooling after $52 \mathrm{Ma}$. The data of $\mathrm{Cibi-}$ cidoides imply that a $\delta^{18} \mathrm{O}$ minimum was reached at approximately $54 \mathrm{Ma}$. If the trend in the planktonic data reflects the climate in the southern high latitudes and intermediate waters were formed there, $\delta^{18} \mathrm{O}$ values of the benthic foraminifer should exhibit the same pattern as the planktonic data. The reduction of the surface to bottom $\delta^{18} \mathrm{O}$ difference with the increase in planktonic values and the decrease in benthic values suggests that intermediate waters in the polar Indian Ocean may have originated at a lower-latitude location in the early Eocene.

\section{Comparison of Benthic Foraminifer Isotopic Records}

Comparison of absolute benthic $\delta^{13} \mathrm{C}$ values from time-series records has been used to infer the source of deep waters in different ocean basins (Miller et al., 1987b; Woodruff and Savin, 1989; Katz and Miller, 1991; Kennett and Stott, 1990). Along their path, deep water masses incorporate $\mathrm{CO}_{2}$ from the dissolution of carbonate and the oxidation of organic matter with low $\delta^{13} \mathrm{C}$ values. Consequently, older waters have higher $\mathrm{CO}_{2}$ content and lower $\delta^{13} \mathrm{C}$ values of bicarbonate than younger waters close to their origin. In Figure $5, \delta^{13} \mathrm{C}$ values of $N$. truempyi from Sites 738 (1200 m paleodepth), 577 (2000 m paleodepth) (Miller et al., 1987b) in the equatorial Pacific, and $690(2000 \mathrm{~m}$ paleodepth) (Kennett and Stott, 1990) in the Weddell Sea area are compared. In general, the records from these three sites exhibit similar trends from the late Paleocene to the early Eocene, although there are greater similarities between the patterns at 


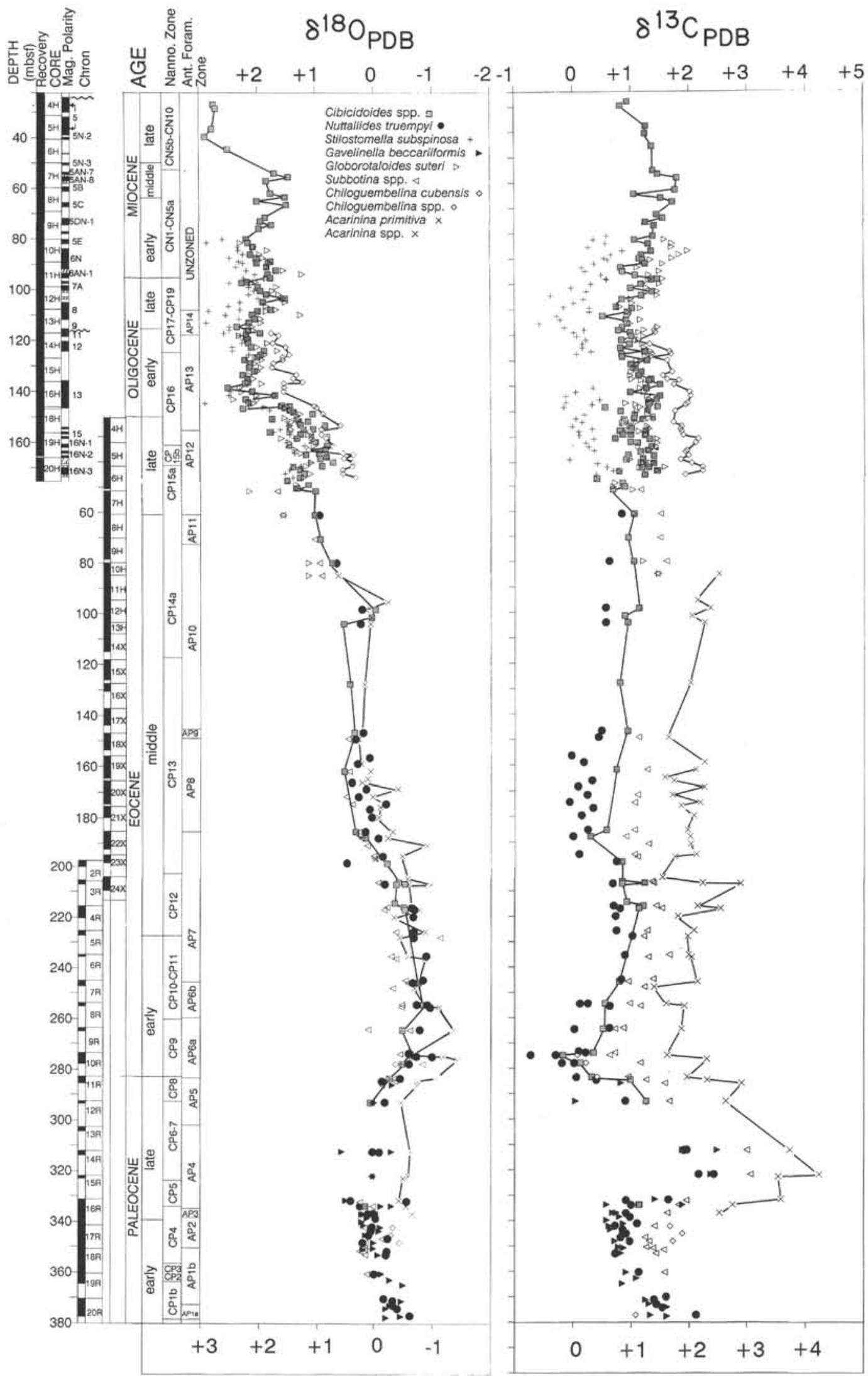

Figure 2. Oxygen and carbon isotope ratios of benthic and planktonic foraminifer taxa from Holes 744B, 744A, $738 \mathrm{~B}$, and $738 \mathrm{C}$ plotted vs. depth in the sediment. For the upper Eocene section of Hole 738B, only isotopic compositions of Cibicidoides spp. are shown. Those of the other taxa analyzed in this study are listed in Table 3. Magnetostratigraphy from Keating (this volume), nannofossil stratigraphy from Wei and Wise (this volume), and planktonic foraminifer stratigraphy from Huber (this volume). 

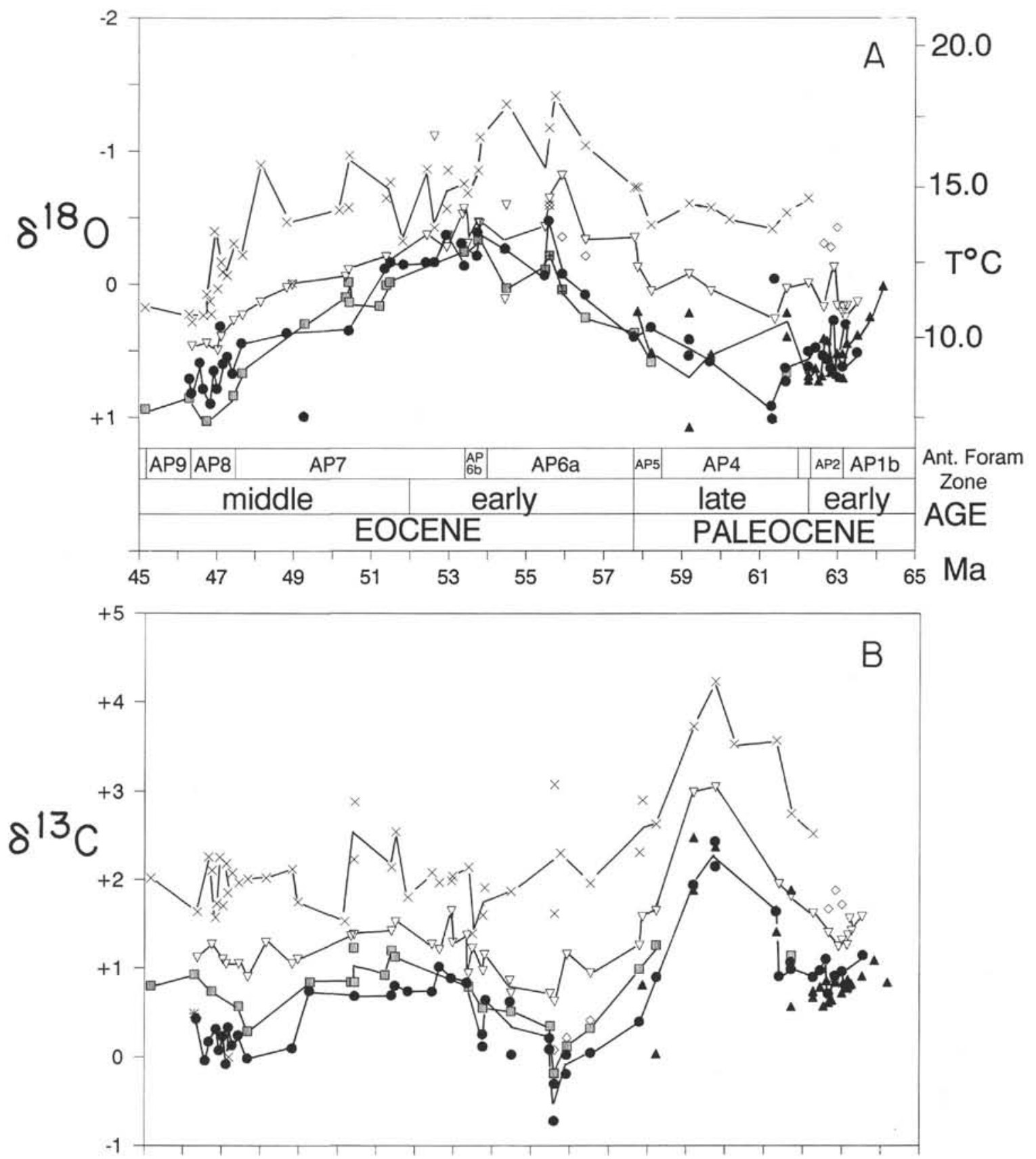

Figure 3. A. Oxygen isotopic compositions of foraminifer taxa from the lower Paleocene to middle Eocene section of Site 738. Adjustments listed in Table 6 have been applied to the benthic foraminifer data. Symbols are as in Figure 2. Paleotemperature estimates using the equation of $O^{\prime}$ Neil et al. (1969) are based on an average seawater $\delta^{18} \mathrm{O}$ value of $-1 \%$. B. Carbon isotopic values. Microfossil zonation boundaries are drawn based on sample datum levels not on absolute ages.

Sites 738 and $577 . \delta^{13} \mathrm{C}$ values at Site 690 prior to $55 \mathrm{Ma}$ were considerably higher than at Sites 577 and 738. After this time, Maud Rise deep waters became more similar to Indian Ocean intermediate waters. $\delta^{13} \mathrm{C}$ values at Site 738 were higher than at Site 577 except between about 62.5 and $61.5 \mathrm{Ma}$ and between 59 and $58 \mathrm{Ma}$. However, the reversals at these times could result from discrepancies in biostratigraphical resolution. Isotopic ratios $\left({ }^{13} \mathrm{C} /{ }^{12} \mathrm{C}\right)$ of Cibicidoides spp. from Site $702(2000 \mathrm{~m}$ paleodepth) (Katz and Miller, 1991) in the South Atlantic are consistently higher than those of Nuttallides at Site 577. This has been cited as evidence for a high-latitude source of intermediate waters in the South Atlantic basins (Katz and Miller, 1991).
Absolute $\delta^{18} \mathrm{O}$ values of $N$. truempyi from Site 690 prior to $55 \mathrm{Ma}$ were in general more enriched than those from Sites 738 and 577 (Fig. 5), probably resulting from colder waters at the Maud Rise produced on the Antarctic margins. Indian Ocean $\delta^{18} \mathrm{O}$ values were lower than those from the Pacific prior to 61 Ma when both locations exhibit the highest values of the Paleocene. Indian and Pacific Ocean records of late Paleocene and early Eocene age prior to $55 \mathrm{Ma}$ appear to have been similar, although small stratigraphic errors in age assignment in this interval can alter this conclusion. During the early Eocene after the maximum warmth, $\delta^{18} \mathrm{O}$ values at Sites 738 and 690 are more similar and on the average lower than those from the Pacific 

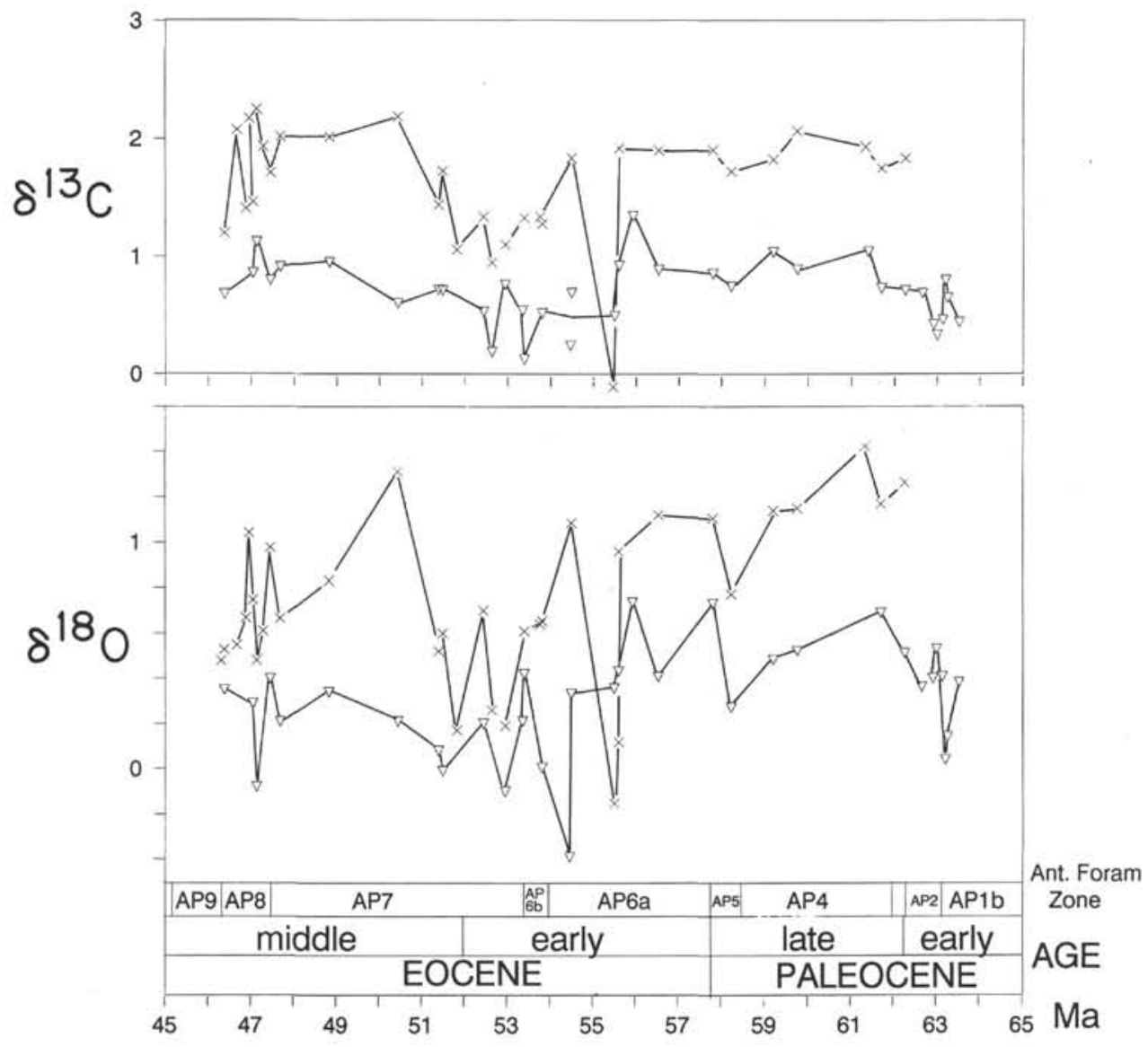

Figure 4. Differences between isotopic compositions of the benthic foraminifer Nuttalides truempyi and the planktonic foraminifers Subbotina spp. and Acarinina spp. from the Paleocene to middle Eocene section of Site 738 . Triangles indicate $\delta^{18} \mathrm{O}_{\text {Nuttallides }}$ minus $\delta^{18} \mathrm{O}_{\text {Subbotina }}$ and $\delta^{13} \mathrm{C}_{\text {Subbotina }}$ minus $\delta^{13} \mathrm{C}_{\text {Nuttallides }}$. The symbol $\mathrm{X}$ represents $\delta^{18} \mathrm{O}_{\text {Nuttallides }}$ minus $\delta^{18} \mathrm{O}_{\text {Acarinina }}$ and $\delta^{13} \mathrm{C}_{\text {Acarinina }}$ minus $\delta^{13} \mathrm{C}_{\text {Nuttallides. }}$.

(Fig. 5). At this time, Indian Ocean $\delta^{13} \mathrm{C}$ values also become more similar to those from the Maud Rise and more enriched than Pacific values. (Similar conclusions are reached by comparing isotopic values of Cibicidoides from Sites 690 and 738 not shown in Fig. 5). These patterns are consistent with the idea proposed by Kennett and Stott (1990) that deep waters at the Maud Rise were produced in the low latitudes in the early Eocene. Maud Rise waters appear to have become as old and warm as those in the Indian and Pacific Oceans at this time. Furthermore, these patterns also suggest that intermediate waters in the Indian Ocean before the early Eocene were different from the Maud Rise waters of probable Antarctic origin. Warm saline waters, perhaps produced in the eastern Tethys, may have flowed at intermediate depths into the high-latitude Indian Ocean even before the early Eocene. Reconstructions of early Paleogene carbon and oxygen isotope distributions at different paleodepths in the Indian Ocean are needed to trace the intermediate water mass to its source.

\section{Latitudinal Gradient in the Early Eocene}

In Figure 6, the mean of $\delta^{18} \mathrm{O}$ values of shallow-dwelling planktonic foraminifer species of Acarinina and Morozovella from lower Eocene deep-sea sediments from Foraminifer Zones P6 to P8 have been plotted against estimated paleolatitudes. These taxa consistently yield the lowest (warmest) $\delta^{18} \mathrm{O}$ values (Shackleton and Boersma, 1981; Shackleton et al., 1985;
Boersma et al., 1987). The shaded area is an envelope through the $\delta^{18} \mathrm{O}$ data of Holocene shallow-dwelling planktonic species (indicated by crosses) from various published sources compiled in Savin et al. (1985). The Holocene data was adjusted by $1 \%$ to compensate for the estimated average $\delta^{18} \mathrm{O}$ composition of sea water in the early Eocene.

The mean of $\delta^{18} \mathrm{O}$ values from Site 738 is close to those of Antarctic Sites 690 and 277. The mean values of these three southern high-latitude sites are in the range of those measured from mid- and low-latitude sites, showing as previously discussed by others, the very shallow or negligible latitudinal gradient of the early Eocene (Savin, 1977; Shackleton and Boersma, 1981). We are assuming the magnitude of the present ${ }^{18} \mathrm{O}$ enrichment of low- and mid-latitude surface sea waters relative to surface sea water in the high-latitudes was the same in the early Eocene. All of early Eocene data points for the mid-latitudes fall within the shaded area defined by the Holocene data, implying that surface water temperatures at these locations were similar to today's. Early Eocene data for the tropics are very limited but the few data points indicate slightly colder tropical surface waters by several degrees than at present. The largest difference between the modern and the early Eocene world is in the southern high latitudes, Holocene shallow-dwelling foraminifers are ${ }^{18} \mathrm{O}$ enriched by several per mils relative to their early Eocene counterparts. Provided that surface seawater $\delta^{18} \mathrm{O}$ in the Antarctic ocean was similar to that of average seawater, as 

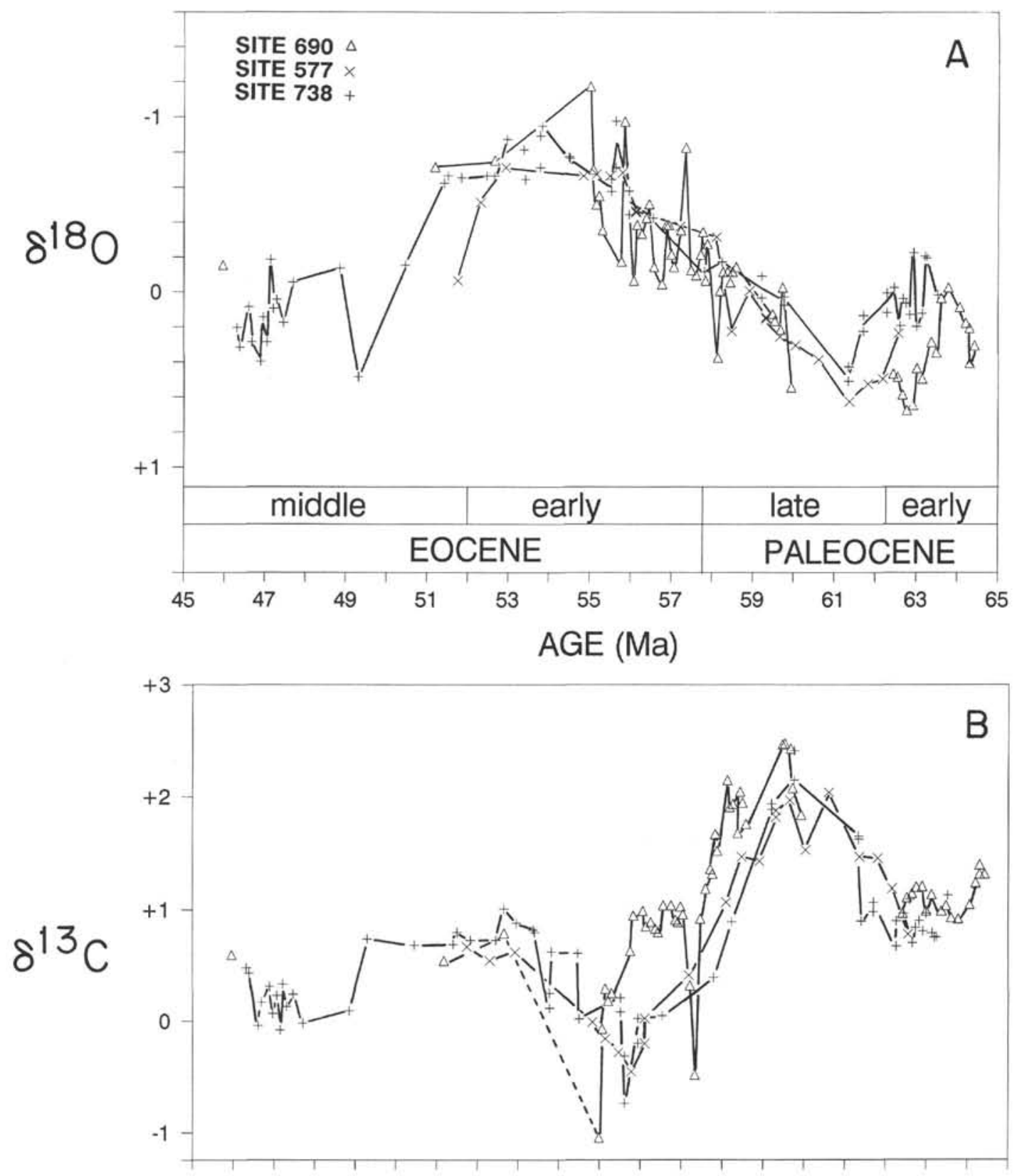

Figure 5. Comparison of (A) oxygen isotopic compositions and (B) carbon isotopic compositions of Nuttallides truempyi from Sites 577, 690, and 738. Isotopic data for Site 577 are from Miller et al. (1987b) and for Site 690 are Kennett and Stott (1990).

it is today (Craig and Gordon, 1965), estimated temperatures based on ${ }^{18} \mathrm{O} /{ }^{16} \mathrm{O}$ ratios of Acarinina from Site 738 were close to $18^{\circ} \mathrm{C}$ during the maximum warmth in the early Eocene (Fig. 3A).

\section{Late Eocene to Early Miocene Records}

\section{Oxygen Isotope Results}

Isotopic compositions of most foraminifer taxa that were analyzed from the upper Eocene to upper Miocene section at Site 744 , and of Cibicidoides spp. from the upper Eocene section at Site 738 are plotted in Figure 2. $\delta^{18} \mathrm{O}$ and $\delta^{13} \mathrm{C}$ values of Cibicidoides spp., Globorotaloides suteri, and Chiloguembelina cubensis from upper Eocene sediments at Site 744 and from con- temporaneous sediments at Site 738 exhibit similar trends and absolute values, allowing easy correlation of these records between sites. For this reason, we restrict our discussion to the records from Site 744 .

The general trend of increasing $\delta^{18} \mathrm{O}$ values in the middle and late Eocene was punctuated at about $39 \mathrm{Ma}(170.57 \mathrm{mbsf})$ by a short reversal when $\delta^{18} \mathrm{O}$ of Cibicidoides spp. and $G$. suteri decreased slightly by about $0.3 \%$ to values close to 0.9 and $1.2 \%$, respectively (Figs. 2 and 7). $\delta^{18} \mathrm{O}$ values of these taxa were higher by about $0.2 \%$ between 38 (159.57 mbsf) and $36.5 \mathrm{Ma}(150.07$ mbsf) and then jumped sharply by $1.15 \% 0$ just above the Eocene/Oligocene boundary. This ${ }^{18} \mathrm{O}$ enrichment occurred in sediments estimated to have been deposited between 36.5 and 35.9 


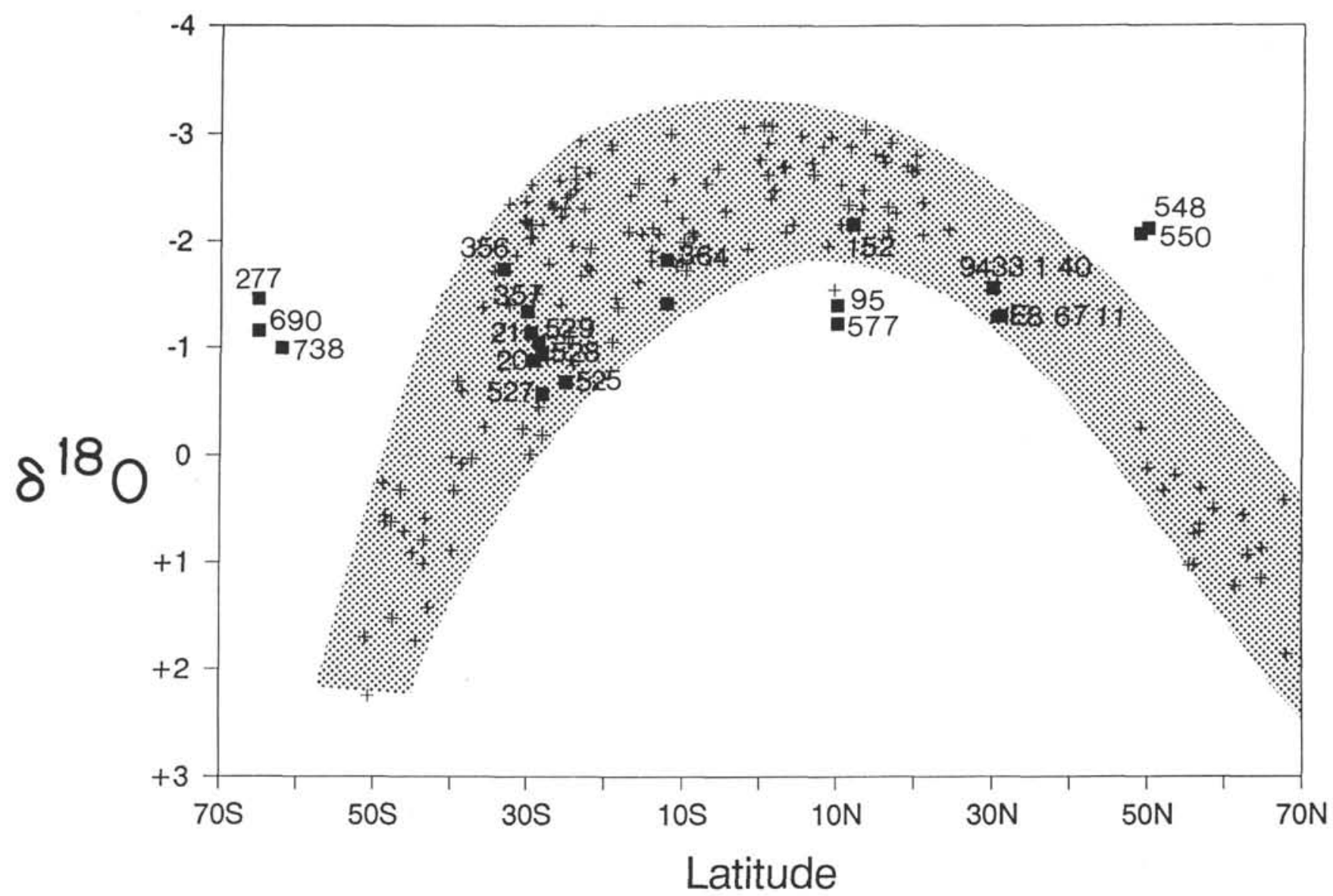

Figure 6. Comparison of latitudinal gradient of $\delta^{18} \mathrm{O}$ values of shallow-dwelling planktonic foraminifers of Holocene age (crosses) and early Eocene age (squares). The shaded area is an envelope through the Holocene data published in Savin et al. (1985). The numbers identify the sites of the early Eocene data which are from Shackleton and Kennett (1975), Shackleton et al. (1985), Boersma et al. (1987), Stott et al. (1990), and this study.

Ma (145.67 mbsf). $\delta^{18} \mathrm{O}$ values of $C$. cubensis increased by $0.5 \%$ at about $38.3 \mathrm{Ma}(164.07 \mathrm{mbsf})$ to values close to $1 \%$ and then, by $1.15 \%$ between 36.5 and $35.9 \mathrm{Ma}$.

After the sharp $\delta^{18} \mathrm{O}$ increase above the Eocene/Oligocene boundary, values of Cibicidoides spp. remained between about 2 and $2.2 \%$ in the early Oligocene, and closer to $2 \% 0$ after 28 Ma (114.17 mbsf) in the late Oligocene (Figs. 2 and 7). In the Miocene, Cibicidoides spp. $\delta^{18} \mathrm{O}$ values increased slightly until about $19 \mathrm{Ma}$ and then varied between 2 and $1.5 \%$ into the early middle Miocene. $\delta^{18} \mathrm{O}$ values of $G$. suteri varied similarly to those of Cibicidoides spp., decreasing to about $2 \%$ in the early Oligocene and to about $1.8 \%$ in the late Oligocene. Early Oligocene ${ }^{18} \mathrm{O} /{ }^{16} \mathrm{O}$ ratios of $C$. cubensis were close to $1.5 \%$.

\section{Carbon Isotope Results}

Cibicidoides spp. $\delta^{13} \mathrm{C}$ values decreased gradually by about $0.9 \%$ in upper Eocene and lower Oligocene sediments until about $36.3 \mathrm{Ma}$ (148.57 mbsf) (Figs. 2 and 7). A $0.8 \%$ rise occurred between 36.3 and $36.15 \mathrm{Ma}$ (147.53 mbsf). Afterward, they decreased gradually in the early Oligocene. Similar $\delta^{13} \mathrm{C}$ patterns as observed near the Eocene/Oligocene boundary at Site 744 have been documented in less detail in benthic records from Sites 253 and 219 in the Indian Ocean (Oberhansli, 1986; Keigwin and Corliss, 1986) and Site 362 in the South Atlantic (Keigwin and Corliss, 1986). The pattern is not recorded in the planktonic records of Site 744 or other sites, suggesting that the change affected only deep waters. The $\delta^{13} \mathrm{C}$ increase in the early Oligocene has been attributed to the enhanced production of young deep waters with the cooling of Antarctica (Oberhansli and Toumarkine, 1985). The data presented here support this hypothesis.

At many sites, surface planktonic and benthic foraminifer $\delta^{13} \mathrm{C}$ decreased from the early to the late Oligocene, and increased from the late Oligocene into the early Miocene (Shackleton and Kennett, 1975; Keigwin and Keller, 1984; Shackleton et al., 1984; Miller and Thomas, 1985). At Site 744, foraminifer $\delta^{13} \mathrm{C}$ values followed this general trend (Figs. 2 and 7).

\section{Surface to Intermediate-Water Isotopic Gradients}

Associated with the decrease in Cibicidoides $\delta^{18} \mathrm{O}$ in the late Eocene at about $39 \mathrm{Ma}(170.57 \mathrm{mbsf})$, the $\delta^{18} \mathrm{O}$ differences between surface and intermediate waters, and between near-surface and intermediate waters at Site 744 were reduced until approximately $35.5 \mathrm{Ma}$ (142.67 mbsf) (Fig. 8 ). These changes occurred while intermediate waters warmed and during subsequent cooling of the entire water column. The thermal stratification between intermediate and near-surface waters was high in the remainder of the Oligocene (Fig. 8). It is not apparent to us what caused the reduction of the stratification in the late Eocene and earliest Oligocene.

The $\delta^{13} \mathrm{C}$ values of intermediate waters became more positive than that of near-surface waters in the earliest Oligocene beginning at $36.15 \mathrm{Ma}(147.53 \mathrm{mbsf})$ and remained so until about 34.02 Ma (130.17 mbsf) (Fig. 8). This is the only instance in the record of the polar Indian Ocean from the southern Kerguelen Plateau when such. a change is apparent. This reversal in the $\delta^{13} \mathrm{C}$ stratification of intermediate-to-surface waters is coincident with the presence of ice-rafted debris in the sediment. Its 

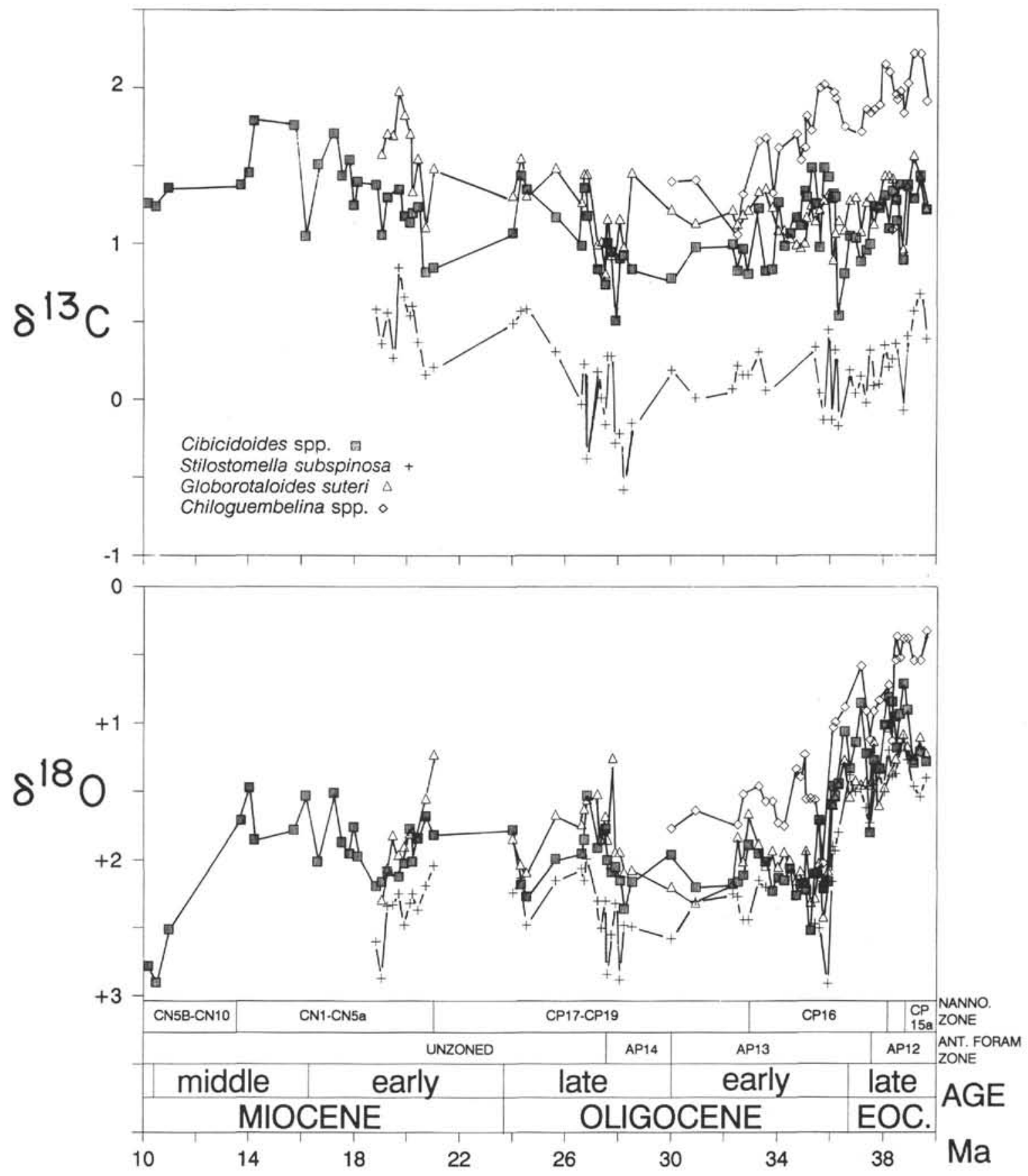

Figure 7. Oxygen and carbon isotopic composition of foraminifer taxa from the upper Eocene to lower Miocene section at Site 744 plotted vs. sediment age. Microfossil zonation boundaries are drawn based on sample datum levels not on absolute ages.

beginning coincides with the first appearance of ice-rafted debris, and it ends close to the level where the last ice-rafted debris was found in sediments of Hole 744A (Fig. 5). We speculate that the high $\delta^{13} \mathrm{C}$ values of Cibicidoides at this time could reflect the increased production of young oxygenated waters on Antarctica.

\section{THE EOCENE/OLIGOCENE TRANSITION}

The Eocene/Oligocene transition at Site 744 (Fig. 9) is characterized by (1) the increase of planktonic foraminifer $\delta^{18} \mathrm{O}$ values in the late Eocene at about $38.4 \mathrm{Ma},(2)$ a gradual decrease in the percent of carbonate beginning in uppermost Eocene sed- iments, (3) an ${ }^{18} \mathrm{O}$ enrichment of $1.15 \%$ just above the Eocene/ Oligocene boundary, and (4) the presence of ice-rafted debris coinciding with the isotopic shift (Fig. 9). This is the first reported simultaneous occurrence of the global $\delta^{18} \mathrm{O}$ shift with ice-rafted debris. Moreover, the events occurring during Eocene/ Oligocene transition in deep-sea sections were recorded in great detail in the section at Site 744. This transition in most deep-sea sections is characterized by hiatuses or breaks in the record near the Eocene/Oligocene boundary (Kennett et al., 1972; Luyendyk and Davies, 1974; Keigwin and Corliss, 1985; Keller et al., 1987). Hiatuses near the boundary are also present in the southern high-latitude sections recovered at Sites 277 and 690 (Shackleton and Kennett, 1975; Kennett and Stott, 1990). At Site 744, 

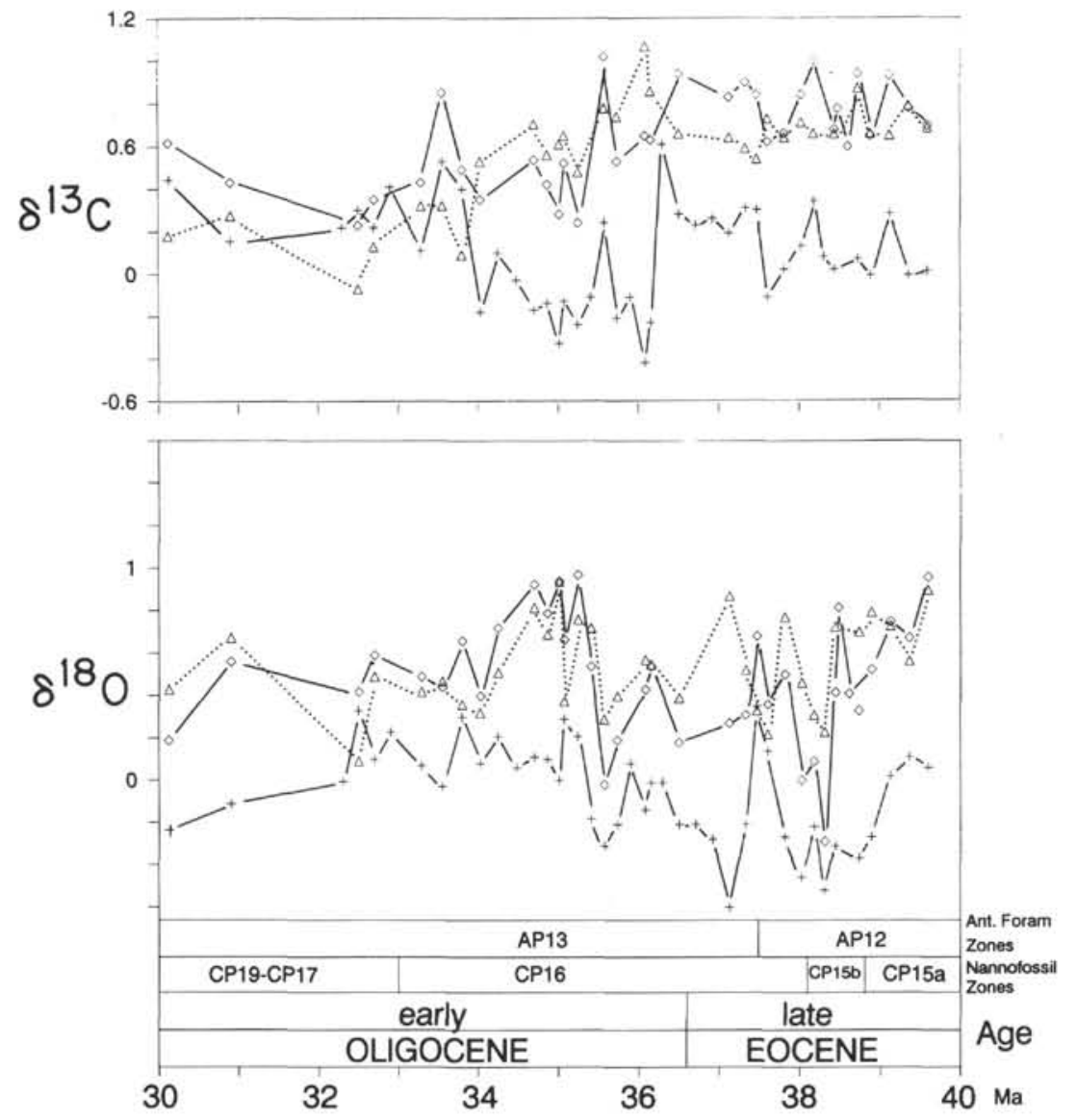

Figure 8. Differences between isotopic compositions of Cibicidoides and planktonic foraminifer species from Site 744. Crosses represent $\delta^{18} \mathrm{O}_{\text {Cibicidoides }}$ minus $\delta^{18} \mathrm{O}_{G \text {. suteri }}$ and $\delta^{13} \mathrm{C}_{G}$. suteri minus $\delta^{13}$ Cibicidoides. Triangles are $\delta^{18} \mathrm{O}_{\text {Cibicidoides }}$ minus $\delta^{18} \mathrm{O}_{C}$. cubensis and

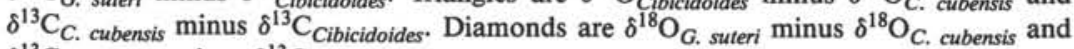
$\delta^{13} \mathrm{C}_{C \text {. cubensis }}$ minus $\delta^{13} \mathrm{C}_{G \text {. suteri }}$.

there is no apparent break in the sedimentary record below or above the Eocene/Oligocene boundary, or above the $\delta^{18} \mathrm{O}$ shift based on evidence from magnetobiostratigraphy (Barron et al., this volume). This permits evaluation of the nature of the change recorded in the $\delta^{18} \mathrm{O}$ shift and conditions before and after.

The $\delta^{18} \mathrm{O}$ increase of $C$. cubensis about 2 m.y. before the boundary $\delta^{18} \mathrm{O}$ shift represents a decline of surface water temperatures by approximately $2^{\circ} \mathrm{C}$. (Ice-volume effect is neglected because the synchronous shift in benthic $\delta^{18} \mathrm{O}$ values is small.) This change was likely significant in affecting the climatic and glacial regime of Antarctica. The cooling of surface waters reflects the northward migration of an ancestral polar front over this area at this time, implying cooling on the continent. The presence of ice-rafted debris in sediments deposited several million years later, requires that the right oceanographic conditions must have developed by then so that icebergs could have travelled to the southern Kerguelen Plateau. Sancetta (1979) proposed based on sedimentological evidence that a convergence or polar front at about $60^{\circ} \mathrm{S}$ had developed in the southern Indian and southwest Pacific Oceans by early Oligocene time, but disappeared in the late Oligocene. At Site 744, the first detectable amount of opaline material consisting of diatoms and radiolarians was found in sediments deposited at $38.5 \mathrm{Ma}$ (167 mbsf) (Ehrmann, this volume) just below this ${ }^{18} \mathrm{O}$ enrichment. Moreover, late Eocene planktonic foraminifer assemblages of low di- versity and taxa with cold water affinity were succeeded by even less diversified assemblages in the early Oligocene (Huber, this volume) indicating a change to a more stressful environment. A marked decline in the abundance of carbonate in the sediment is indicative either of changes in the supply at the surface or dissolution at the seafloor. A decrease in the abundance of carbonate to values below $90 \%$ was noted between Sample 119-744A-18H-2, 95-100 cm, and Sample 119-744A-18H-1, 95-100 cm (Table 7, Fig. 9). Below this level, carbonate content in upper Eocene sediments is greater than $92 \%$. Moreover, siliceous microfossils increased in abundance in sediments between these samples, and remained dominant to the upper part of Core $119-744 \mathrm{~A}-16 \mathrm{H}$. The presence of solution features in planktonic and benthic foraminifer tests beginning in Sample 119-744A-18H-1, 95-100 cm, lends support to the argument for increased dissolution at the seafloor.

The foraminifer ${ }^{18} \mathrm{O}$ enrichment of $1.15 \% 0$ is observed in sediments estimated to have been deposited between 36.5 and 35.9 $\mathrm{Ma}$, which gives a duration of at least $0.6 \mathrm{~m}$.y. to this event (Fig. 9). The shift begins in sediments at the top of magnetic reversal 13 and ends at the base of Chron 13N (Fig. 2). This global increase in planktonic and benthic foraminifer $\delta^{18} \mathrm{O}$ values has been attributed to both a decrease in high-latitude temperatures and an increase in ice volume (Keigwin and Corliss, 1986; Miller et al., 1987b; and others). At Site 744, ice rafted-debris occur in sediments deposited during the $\delta^{18} \mathrm{O}$ shift and at its maximum 


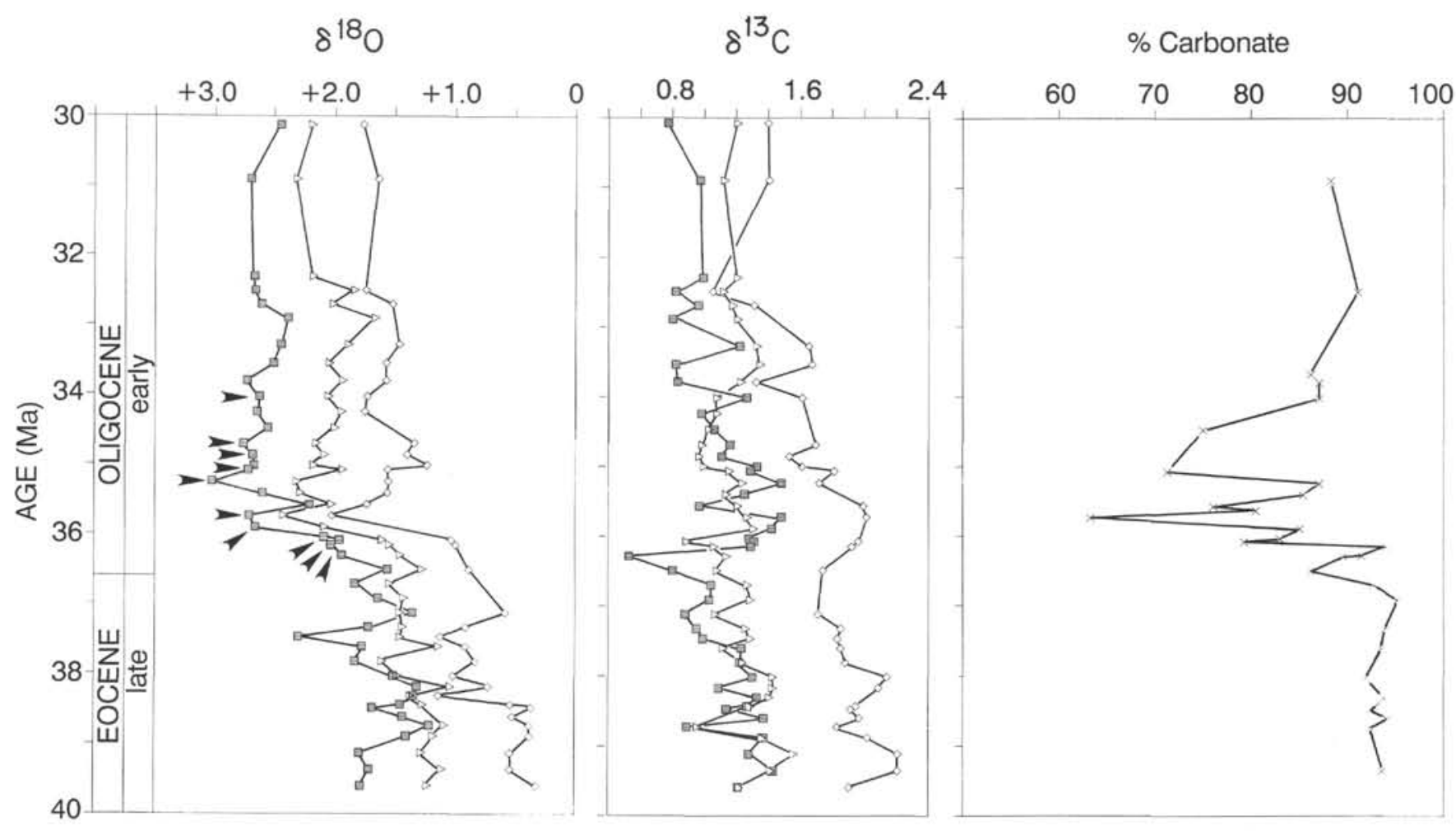

Figure 9. Oxygen and carbon isotopic compositions of foraminifer taxa and percent carbonate from the upper Eocene to lower Oligocene section of Site 744. The arrows in the $\delta^{18} \mathrm{O}$ plot point to foraminifer samples with ice-rafted debris. Oxygen isotope ratios of Cibicidoides have been adjusted by the amount listed in Table 6. Carbonate data are tabulated in Table 7; symbols are as in Figure 7.

(Fig. 9), directly linking the isotopic change to glaciation in Antarctica. The presence of ice-rafted debris on the southern Kerguelen Plateau implies that these sediments must have been delivered there by icebergs. Debris-laden icebergs result from calving of ice streams and outlet glaciers at sea level (Anderson, 1985). Icebergs of this type can also be produced from the calving of ice shelves containing sediment frozen at the bottom (Hambrey, this volume). Production of icebergs by either mode indicates that a substantial mass of ice must have been present on Antarctica. Moreover, ice-rafted debris are found in sediments deposited before the maximum ${ }^{18} \mathrm{O}$ enrichment. This pattern of sedimentation and $\delta^{18} \mathrm{O}$ shows that an ice cap had begun to form in East Antarctica several million years before $36 \mathrm{Ma}$ when the highest $\delta^{18} \mathrm{O}$ values were measured, and that glacial build-up occurred over less than $1 \mathrm{~m}$.y. The effect of ice buildup on the oxygen isotopic composition of seawater should be greater during the later stages of ice-cap growth when the isotopic composition of precipitated snow is more ${ }^{16} \mathrm{O}$ enriched relative to that of seawater. Therefore, the maximum ${ }^{18} \mathrm{O}$ enrichment may be equated with the existence of a large ice sheet. The sharp increase in Cibicidoides spp. $\delta^{13} \mathrm{C}$ values coinciding with the first appearance of ice-rafted debris may reflect the production of cold dense waters at the Amery Shelf during sea ice formation, or perhaps underneath an ice shelf. Evidence for the vigorous production of bottom waters on Antarctica in the earliest Oligocene is also found in the difference between $\delta^{18} \mathrm{O}$ of benthic foraminifers from low-latitude and high-latitude sites, which is discussed below.

At Site 744 , ice-rafted debris was found within the zone of maximum ${ }^{18} \mathrm{O}$ enrichment (Erhman, this volume) and for about 2 m.y. after, whereas Cibicidoides spp. $\delta^{18} \mathrm{O}$ values remained high during at least the early and late Oligocene (Fig. 9). The presence of ice-rafted debris in the Kerguelen Plateau sediments indicates very dynamic glacial processes on Antarctica either during the formation or retreat of an ice cap. At these times more debris-laden icebergs should be produced (Anderson, 1985). Oceanographic factors must also play a role in determining the sites of accumulation in the deep sea. The disappearance of icerafted debris in lower Oligocene sediments may indicate that the large ice cap had a short life in the earliest Oligocene and soon receded from the coast. Conversely, oceanographic factors could have prevented the accumulation of debris in the southern Kerguelen Plateau. Nevertheless, measured Oligocene Cibicidoides spp. $\delta^{18} \mathrm{O}$ values of $2 \%$ or more must reflect a combination of a small ice effect and lower water temperatures at Site 744. This small ice cap grew to large proportions in the earliest late Oligocene when Cibicidoides $\delta^{18} \mathrm{O}$ values at Site 689 were about $3.5 \%$ (Kennett and Stott, 1990) and foraminifer $\delta^{18} \mathrm{O}$ values in lowlatitude sites recorded synchronous enrichments (Keigwin and Keller, 1984; Miller and Thomas, 1985). Unfortunately, a hiatus spans the length of these events at Site 744 (Barron et al., this volume).

\section{IMPLICATIONS FOR OLIGOCENE DEEP WATER ORIGIN AND CIRCULATION}

It has been proposed that the establishment of the thermohaline circulation and the psychrosphere occurred in the early Oligocene with the refrigeration of Antarctica and the formation of sea ice (Shackleton and Kennett, 1975; Kennett and Shackleton, 1976). More recently, Kennett and Stott (1990) have suggested that deep water circulation in the Oligocene oceans was driven by temperature as well as salinity differences from at least two sources located in the high and low latitudes. Based on inversions of benthic foraminifer isotopic data from Sites 689 and 690 closely situated at different depths in the Weddell Sea area, they propose that Oligocene water masses at shallow depths 
Table 7. Percent carbonate abundance at selected levels of Hole 744A.

\begin{tabular}{|c|c|c|}
\hline $\begin{array}{l}\text { Core, section, } \\
\text { interval }(\mathrm{cm})\end{array}$ & $\begin{array}{l}\text { Depth } \\
\text { (mbsf) }\end{array}$ & $\begin{array}{c}\text { Carbonate } \\
(\%)\end{array}$ \\
\hline $13 \mathrm{H}-\mathrm{CC}$ & 118.20 & ${ }^{a} 88.3$ \\
\hline $14 \mathrm{H}-2,80-82$ & 120.50 & ${ }^{\mathrm{a}} 91.1$ \\
\hline $14 \mathrm{H}-7,15-17$ & 127.35 & $\mathrm{a} 87.1$ \\
\hline $14 \mathrm{H}-\mathrm{CC}$ & 127.55 & ${ }^{\mathrm{a}} 86.2$ \\
\hline $15 \mathrm{H}-1,60-62$ & 128.30 & ${ }^{a} 87.1$ \\
\hline $15 \mathrm{H}-3,105-106$ & 131.75 & ${ }^{\mathrm{a}} 75.0$ \\
\hline $15 \mathrm{H}-\mathrm{CC}$ & 137.40 & ${ }^{\mathrm{a}} 71.3$ \\
\hline $16 \mathrm{H}-1,92-93$ & 138.12 & $a_{87.1}$ \\
\hline $16 \mathrm{H}-1,89-94$ & 138.12 & $b_{87.7}$ \\
\hline $16 \mathrm{H}-2,95-100$ & 139.67 & $b_{85.4}$ \\
\hline $16 \mathrm{H}-3,95-100$ & 141.17 & $b_{76.1}$ \\
\hline $16 \mathrm{H}-4,95-100$ & 142.67 & $b_{80.5}$ \\
\hline $16 \mathrm{H}-4,145-150$ & 143.15 & $\mathrm{a} 63.3$ \\
\hline $16 \mathrm{H}-5,95-100$ & 144.17 & $b_{85.0}$ \\
\hline $16 \mathrm{H}-6,95-100$ & 145.67 & $\mathrm{~b}_{83.0}$ \\
\hline $16 \mathrm{H}-\mathrm{CC}$ & 146.90 & ${ }^{a} 79.3$ \\
\hline $16 \mathrm{H}-\mathrm{CC}$ & 146.90 & ${ }^{b} 60.3$ \\
\hline $17 \mathrm{H}-1,28-33$ & 147.01 & $b_{93.9}$ \\
\hline $17 \mathrm{H}-\mathrm{CC}$ & 147.17 & 91.5 \\
\hline $17 \mathrm{H}-\mathrm{CC}$ & 147.53 & ${ }^{b} 86.2$ \\
\hline $18 \mathrm{H}-1,91-92$ & 148.51 & ${ }^{2} 89.6$ \\
\hline $18 \mathrm{H}-1,95-100$ & 148.57 & $b_{86.2}$ \\
\hline $18 \mathrm{H}-2,95-100$ & 150.07 & $b_{92.8}$ \\
\hline $18 \mathrm{H}-3,95-100$ & 151.57 & $b_{95.1}^{32.0}$ \\
\hline $18 \mathrm{H}-5,95-100$ & 154.57 & $b_{93.9}$ \\
\hline $18 \mathrm{H}-\mathrm{CC}$ & 157.10 & 93.5 \\
\hline $19 \mathrm{H}-2,95-100$ & 159.57 & $b_{91.9}$ \\
\hline $19 \mathrm{H}-4,145-150$ & 163.05 & $a_{93.8}$ \\
\hline $19 \mathrm{H}-5,134-135$ & 164.44 & $a_{92.9}$ \\
\hline $19 \mathrm{H}-6,95-100$ & 165.57 & $b_{92.4}$ \\
\hline $19 \mathrm{H}-\mathrm{CC}$ & 165.60 & $a_{94.1}$ \\
\hline $20 \mathrm{H}-1,95-100$ & 167.57 & $b_{92.4}$ \\
\hline $20 \mathrm{H}-4,97-98$ & 172.02 & ${ }^{2} 93.6$ \\
\hline $20 \mathrm{H}-\mathrm{CC}$ & 176.32 & 95.1 \\
\hline
\end{tabular}

in the southern South Atlantic Ocean as well as bottom waters of the ocean basins originated in Antarctica, whereas intermediate and deep water masses had a Tethyan source. To investigate this possibility we have compared the $\delta^{18} \mathrm{O}$ record of Cibicidoides from Sites 738, 744 and 689 to those of the same species from low- and mid-latitude Sites 574 and 77 (2650 and $2900 \mathrm{~m}$ paleodepth), and 522 ( $2700 \mathrm{~m}$ paleodepth), and of mixed benthic foraminifer species from Sites 525 (1400 m paleodepth), 527 (3000 $\mathrm{m}$ paleodepth), and 529 (2300 m paleodepth). The locations of these sites are shown in Figure $10 .\left(\delta^{18} \mathrm{O}\right.$ values from the last three sites, which have been adjusted for disequilibrium effects by Shackleton et al. (1984), have been adjusted to reflect the composition of Cibicidoides by subtracting $0.5 \%$.) These data have been plotted in Figure 11 using the published ages based on the chronology of Berggren (1985a, 1985b). These are the most complete benthic foraminifer records available for the Oligocene and those from the greatest water depth in the $\mathrm{Pa}$ cific. Unfortunately, there are no middle or late Eocene records of intermediate and deeper waters for the Pacific that constituted most of the world's oceans at the time.

Middle and late Eocene $\delta^{18} \mathrm{O}$ values from Site 527 are not very different from those of Sites 738, 744, and 689 in the middle and late Eocene (Fig. 11). In the early Oligocene beginning at the ${ }^{18} \mathrm{O}$ enrichment, there is great similarity among $\delta^{18} \mathrm{O}$ values of the low-latitude sites, but these are on the average about $0.5 \%$ lower than those of Sites 744 and 689 . For example, averages for the early Oligocene interval $35-30 \mathrm{Ma}$ are: $1.57 \pm 0.17$, $\mathrm{N}=28$ for Site $522 ; 1.58 \pm 0.17, \mathrm{~N}=13$ for Site $529 ; 1.60 \pm$ $0.30, \mathrm{~N}=9$ for Site $574 ; 1.57 \pm 0.23, \mathrm{~N}=21$ for Site $77 ; 2.12$ $\pm 0.12, \mathrm{~N}=15$ for Site 744 ; and $2.26 \pm 0.18, \mathrm{~N}=22$ from
Site 689. This difference persisted in the late Oligocene and into the Miocene (Fig. 11). In the early Oligocene (30 to $35 \mathrm{Ma}$ ), average $\delta^{13} \mathrm{C}$ values are: $0.77 \pm 0.24, \mathrm{~N}=24$ at Site $522 ; 0.47 \pm$ 0.23 at Site $574 ; 0.65 \pm 0.23, N=26$ at Site $77 ; 0.93 \pm 0.27, N$ $=10$ at Site 744; and $0.7 \pm 0.19, \mathrm{~N}=22$ for Site 689. Unlike today, Pacific deep waters were not ${ }^{13} \mathrm{C}$ depleted relative to other basins. In the late Oligocene, average $\delta^{13} \mathrm{C}$ values of equatorial Pacific deep waters did not change, but the difference between intermediate waters in the polar Indian Ocean $(1.08 \pm 0.24, \mathrm{~N}$ $=9)$ and deep waters in the South Atlantic $(1 \pm 0.39, \mathrm{~N}=13)$ decreased. North Atlantic Deep Water (NADW) produced intermittently in the Oligocene (Miller and Tucholke, 1983; Miller and Fairbanks, 1983) increased $\delta^{13} \mathrm{C}$ of bicarbonate in the South Atlantic. However, we note that $\delta^{13} \mathrm{C}$ values of deep waters in the South Atlantic are higher than those in the equatorial Pacific in the early Miocene when there is no evidence of NADW production (Woodruff and Savin, 1989).

Calculated $\delta^{18} \mathrm{O}$ values of carbonate precipitated in equilibrium with modern deep waters (Ostlund, 1987; O'Neil et al., 1969) with those at the Oligocene paleodepth of the Pacific and Atlantic sites are very close, but about $0.4 \% 0$ different from that precipitated near Site 744 . Therefore, the Oligocene isotopic differences are not unlike those observed today. Site 744 is currently under the circumpolar deep water that is colder by a few degrees than Pacific and South Atlantic deep waters. By the early Oligocene, a prototype of the modern Antarctic water mass had likely developed with the opening of an intermediate water connection between Antarctica and Australia and possible deep water circulation across the Drake Passage (Le Brecque and Rabinowitz, 1977). These oceanographic changes may account for the observed increase in isotopic differences relative to the late Eocene. An alternative but less probable scenario is that the flow of Antarctic bottom waters in southern high-latitude oceans was restricted to the ocean basins close to the continent by topographical features. But the ridges that divided the Indian Ocean into separate basins below $2000 \mathrm{~m}$ had subsided by the Oligocene (Sclater et al., 1977).

Unfortunately, the available isotopic data are insufficient to reconstruct oceanic circulation patterns and to either prove or disprove the low-latitude origin of deep waters in the South Atlantic and Pacific Oceans in the Oligocene. For the relatively warm early Miocene, Woodruff and Savin (1989) have presented isotopic evidence for the formation of the southern oceans intermediate waters in the Indian Tethys, whereas deep waters were of Antarctic origin. The Miocene data also suggest that a water mass of Antarctic origin progressively filled the bottom and deep water depths of the equatorial and North Pacific Oceans after $16 \mathrm{Ma}$ (see Figs. 10 and 11 in Woodruff and Savin (1989)). A reduction in the $\delta^{13} \mathrm{C}$ difference between South Atlantic and Pacific deep waters also occurred at about this time. Deep and intermediate waters in the Pacific may have had another source before this time, probably located in the low latitudes, or were a mixture of waters derived from low- and highlatitude sources. We suggest that the production of cold dense waters on the Antarctic margins increased in the early Oligocene when there were competing sources for deep water formation, but was only greatly intensified in the middle Miocene with the build-up of Antarctic continental ice when additional sources disappeared or decreased.

The intensification of Antarctic bottom water production in the early Oligocene appears to be linked to the presence of widespread hiatuses in the southwestern and southeastern Indian and southwestern Pacific Oceans (Kennett et al., 1972; Luyendyk and Davies, 1974; Keller et al., 1987). Oligocene sedimentation, however, proceeded uninterrupted in the northwestern Indian Ocean. In the rest of the Indian Ocean, hiatuses are found in sequences deposited at shallow and deep-water depths, a pat- 


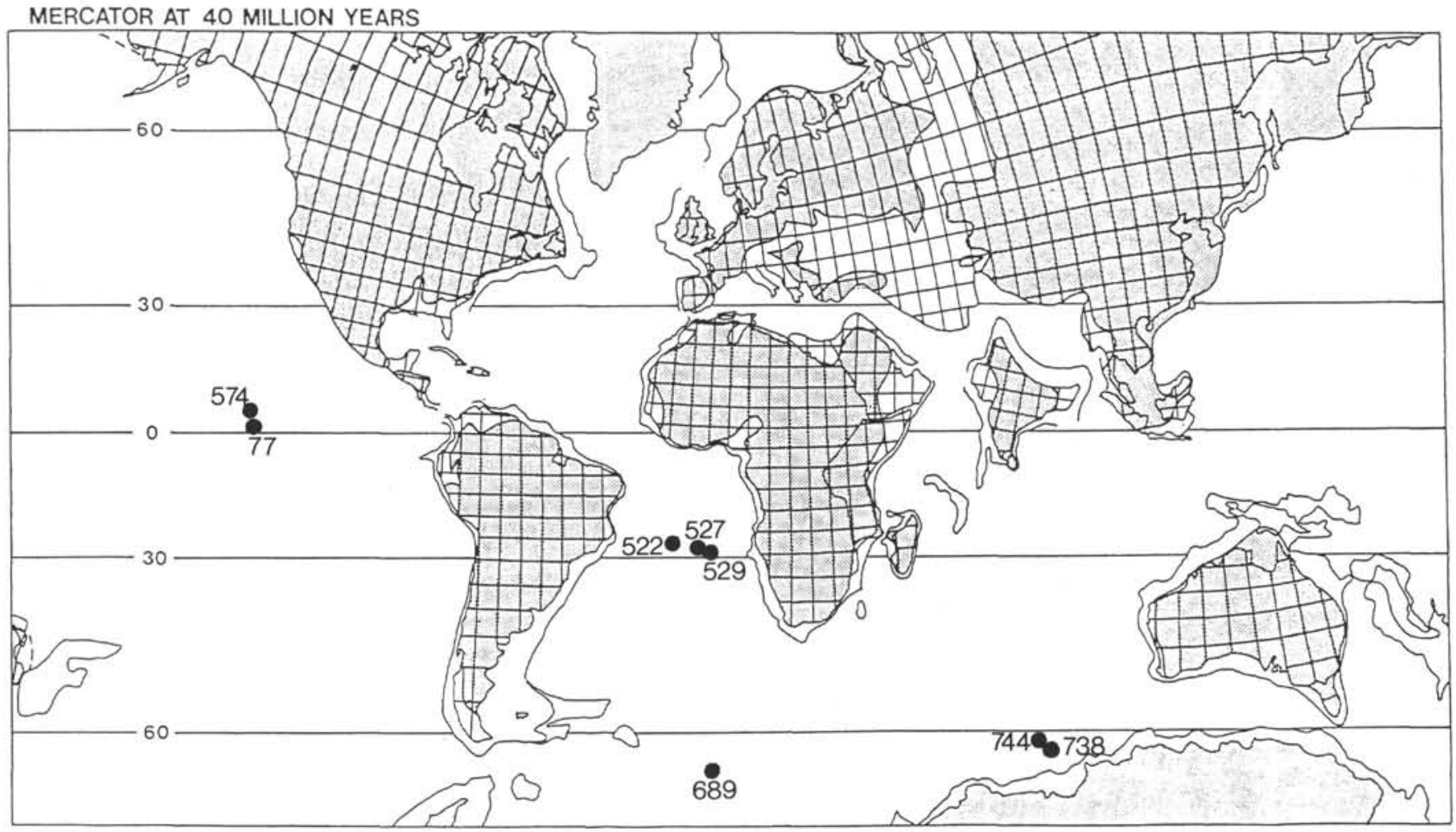

Figure 10. Locations of deep-sea sites from which data are plotted in Figure 11. Map from Barron et al. (1981).

tern which has been interpreted indicative of strong surface and deep water currents (Luyendyk and Davies, 1974; Davies and Kidd, 1977). Near the Eocene/Oligocene boundary, the centers of deep-sea carbonate deposition shifted to the equatorial $\mathrm{Pa}$ cific and South Atlantic Oceans where the carbonate compensation depth (CCD) dropped sharply (Van Andel et al., 1977; Hsü et al., 1984; Moore et al., 1984). The lowering of the CCD in these basins has been attributed to the influx of young oxygenated bottom waters of Antarctic origin (Berger, 1973). An alternative but not unrelated explanation is that increased productivity of calcareous plankton in the open oceans was caused by the higher continental fluxes of nutrients during low sea stands (Van Andel et al., 1977) which resulted from continental glaciation. It is possible that such fractionation in the chemistry of bottom waters in these basins was caused by differences in water mass sources. More detailed isotopic study of Oligocene sediments from different ocean basins are needed to reconstruct the oceanography of this interesting epoch.

\section{CONCLUSIONS}

Oxygen and carbon isotope records of foraminifer samples from Sites 738 and 744 on the southern Kerguelen Plateau provide insights into climatic and oceanographic events on the Antarctic Indian Ocean from the early Paleocene to the Miocene.

Intermediate and surface-water temperatures inferred from oxygen isotopic compositions of foraminifers were relatively low in the early Paleocene. The surface-to-bottom thermal stratification was high at this time. After increasing by about $6^{\circ} \mathrm{C}$ (1.5\% change) from an early late Paleocene minimum (about $8^{\circ} \mathrm{C}$ ), the highest temperatures of intermediate waters in the polar Indian Ocean were recorded in the period from approximately 56 to $54 \mathrm{Ma}\left(\sim 13^{\circ} \mathrm{C}\right)$. Waters at these depths cooled markedly after about $52 \mathrm{Ma}$. Synchronous with the maximum warmth, carbon isotopic compositions of benthic foraminifers reached the lowest values of the Paleogene. Surface and near- surface waters were also warmest at about $56 \mathrm{Ma}\left(\sim 18^{\circ} \mathrm{C}\right.$ for surface waters) and subsequently declined gradually during the early and middle Eocene. The decrease of the isotopic differences between surface/near-surface to intermediate waters from 56 to $52 \mathrm{Ma}$, while surface waters cooled, suggests that intermediate waters may not have formed in Antarctica at that time.

Comparison of benthic foraminifer records from Site 738 with those from Pacific Site 577 and southern South Atlantic Site 690 shows that prior to $56 \mathrm{Ma}$, intermediate waters in the Indian and Pacific Oceans were warmer and ${ }^{13} \mathrm{C}$ depleted relative to intermediate waters in the Weddell Sea. Weddell Sea waters were likely of Antarctic origin. In the remainder of the early Eocene, the records from these three sites are similar. Although this similarity could be interpreted to reflect a single source for intermediate waters in these basins, the source could have been located at any latitude. Kennett and Stott (1990) have proposed the low-latitude formation of intermediate waters at Site 690 during most of the Eocene. However, the data available at present are insufficient to resolve the issue of the source of intermediate waters in the Indian Ocean.

Most of the cooling of the polar Indian Ocean during the Paleogene occurred in the middle and late Eocene. Oxygen isotopic compositions of benthic and planktonic foraminifers increased by about $1 \%$ from the earliest middle Eocene to about $47 \mathrm{Ma}$. The upper middle Eocene record of Site 738 was sparsely sampled at this time but oxygen isotopic values appear to have increased by nearly $1 \% 0$ within this interval.

The global ${ }^{18} \mathrm{O}$ enrichment just above the Eocene/Oligocene boundary is recorded at Site 744 (about $1.1 \%$ change) coincident with the presence of a considerable amount of ice-rafted debris in the sediments. This simultaneous occurrence directly links the isotopic shift to significant glaciation in Antarctica. In fact, a 700-m-thick package of lower Oligocene diamictites was recovered at Site 739 in Prydz Bay, which implies that the Lambert Glacier/Amery Ice Shelf Complex was grounded $140 \mathrm{Km}$ beyond the edge of the present ice sheet (Barron et al., this vol- 

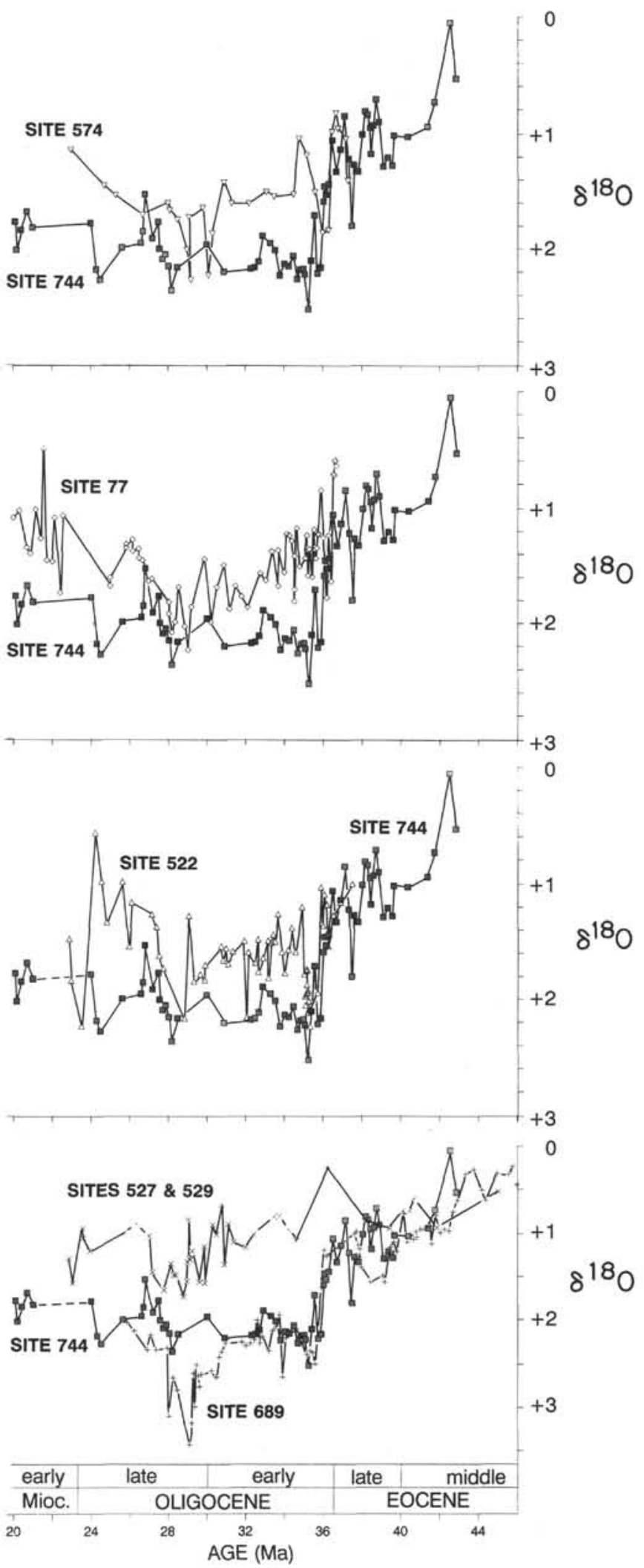

Figure 11. Comparison of oxygen isotope records of species of Cibicidoides and mixed benthic (as noted) from several deep-sea sites. The adjustment applied to the mixed benthic species data from Sites 527 and 529 (from Shackleton et al., 1984) is discussed in the text. Data sources are: Keigwin and Keller (1984) for Site 77; Miller and Thomas (1985) for Site 574; Miller et al. (1988) for Site 522; Kennett and Stott (1990) for Site 689 . ume). The isotopic increase occurred gradually in sediments dated at 36.5-35.9 Ma, whereas ice-rafted debris was deposited beginning at $36.1 \mathrm{Ma}$, when Cibicidoides values were about $1.5 \%$. This pattern indicates that extensive Antarctic glaciation occurred before seawater $\delta^{18} \mathrm{O}$ was significantly affected by ice-volume. The presence of diamictites and glacial marine sediments of latest middle Eocene to late Eocene age recovered at Prydz Bay Site 742 (Barron et al., this volume) corroborates this conclusion.

The major pulse of early Oligocene glaciation in East Antarctica was preceded by a $2^{\circ} \mathrm{C}$ decline in surface water temperatures at about $38.4 \mathrm{Ma}$, which we infer to reflect the migration of a polar front over the southern end of the Kerguelen Plateau. A notable increase in the relative abundance of biogenic silica (Erhman, this volume) occurred at about the same time as the late Eocene cooling. Further cooling and establishment of a polar front occurred in the early Oligocene as evidenced by a further increase of biogenic siliceous sediments (Barron et al., this volume). The decrease of surface-water temperatures in the late Eocene may have been significant in affecting the glacial regime in Antarctica leading to the major glaciation episode in the early Oligocene. It is possible that with the same amount of precipitation over the continent, lower temperatures enhanced the accumulation of ice.

Cibicidoides $\delta^{18} \mathrm{O}$ values close to $2 \%$ imply that extensive glacial conditions persisted in Antarctica during the Oligocene. This is supported by evidence from diamictite sequences of early Oligocene age in Prydz Bay (Barron et at., this volume) and late Oligocene age in the Ross Sea (Barrett, 1989). Although the volume of ice varied, $\delta^{18} \mathrm{O}$ values suggest that Antarctica was never free of ice during the Oligocene.

The production of cold dense waters in the Antarctic margins was intensified with continental cooling and glaciation in the early Oligocene. Comparison of benthic foraminifer records from low- and high-latitude sites shows relatively uniform $\delta^{18} \mathrm{O}$ values in the Eocene. After the global ${ }^{18} \mathrm{O}$ enrichment in the early Oligocene, $\delta^{18} \mathrm{O}$ values from low-latitude sites decreased by about $0.5 \%$ (to about $1.5 \%$ ), while values from high-latitude Sites 689 and 744 remained close to $2 \%$. This $\delta^{18} \mathrm{O}$ difference between sites at these two locations persisted during the Oligocene and in the early Miocene. We propose that these differences reflect the flow of cold dense waters produced during sea ice formation to the sites close to Antarctica, whereas lower-latitude sites were bathed by more locally produced waters.

\section{ACKNOWLEDGMENTS}

The authors acknowledge the help of Bradley Opdyke with the isotopic analyses. K.C. Lohmann generously provided the use of the laboratory facilities, and the Ocean Drilling Program provided the samples. Janice Pappas and Linda Koch gave technical assistance. We thank Gerta Keller, Samuel Savin, and an anonymous reviewer for comments that improved the manuscript. This research was supported in part by NSF grants OCE8800049 and DPP-8816833 to E. Barrera.

\section{REFERENCES}

Anderson, J. B., 1985. Antarctic Glacial Marine Sedimentation: A Core Workshop: Geol. Soc. Am. Quat. Geol. and Geomorphol. Div.

Axelrod, D. I., 1984. An interpretation of Cretaceous and Tertiary biota in polar regions. Palaeogeogr., Palaeoclimatol., Palaeoecol., 45: 105-147.

Barrett, P. J. (Ed.), 1989. Antarctic Cenozoic history from the CIROS-1 drillhole, McMurdo Sound. DSIR Bull., 245.

Barron, E. J., Harrison, C.G.A., Sloan, II, J. L., Hay, W. W., 1981. Paleogeography, 180 million years ago to the present. Eclogae. Geol. Helv., 74:443-470.

Barron, J., Larsen, B., et al., 1989. Proc. ODP, Init. Repts., 119: College Station, TX (Ocean Drilling Program). 
Belanger, P. E., Curry, W. B., and Matthews, R. K., 1981. Core-top evaluation of benthic foraminiferal isotopic ratios for paleoceanographic interpretations. Palaeogeogr., Palaeoclimatol., Palaeoecol., 33:205-220.

Berger, W. H., 1973. Cenozoic sedimentation in the eastern tropical Pacific. Geol. Soc. Am. Bull., 84:1941-1954.

Berggren, W. A., Kent, D. V., and Flynn, J. J., 1985. Jurassic to Paleogene: Part 2. Paleogene geochronology and chronostratigraphy. In Snelling, N. J. (Ed.), The Chronology of the Geological Record. Geol. Soc. London Mem., 10:141-195.

Berggren, W. A., Kent, D. V., and Van Couvering, J. A., 1985. The Neogene: Part 2. Neogene geochronology and chronostratigraphy. In Snelling, N. J. (Ed.), The Chronology of the Geological Record. Geol. Soc. London Mem., 10:211-260.

Boersma, A., Premoli Silva, I., and Shackleton, N. J., 1987. Atlantic Eocene planktonic foraminiferal paleohydrographic indicators and stable isotope paleoceanography. Paleoceanography, 2:287-331.

Brass, G. W., Southam, J. R., and Peterson, W. H., 1982. Warm saline bottom water in the ancient ocean. Nature, 296:620-623.

Chamberlin, T. C., 1906. On a possible reversal of deep-sea circulation and its influence on geologic climates. J. Geol., 14:363-373.

Craig, H., 1957. Isotopic standards for carbon and oxygen correction factors for mass spectrometric analysis of $\mathrm{CO}_{2}$. Geochim. Cosmochim. Acta, 12:133-149.

Craig, H., and Gordon, L., 1965. Deuterium and oxygen-18 variation in the ocean and marine atmosphere. Univ. Rhode Island Occas. Publ., $3: 277-374$.

Davies, T. A., and Kidd, R. B., 1977. Sedimentation in the Indian Ocean through time. In Heirtzler, J. R., Bolli, H. M., Davies, T. A., Saunders, J. B., and Sclater, J. G. (Eds.), Indian Ocean Geology and Biostratigraphy. Am. Geophys. Union, 61-86.

Detrick, R. S., Sclater, J. G., and Thiede, J., 1977. The subsidence of aseismic ridges. Earth Planet. Sci. Lett., 34:185-198.

Emiliani, C., 1956. On paleotemperatures of Pacific bottom waters. Science, 123:460-461.

Estes, R., and Hutchinson, J. H., 1980. Eocene lower vertibrates from Ellesmere Island, Canadian Arctic Archipelago. Palaeogeogr., Palaeoclimatol., Palaeoecol., 30:325-347.

Garrison, R. E., 1981. Diagenesis of oceanic carbonate sediments: a review of the DSDP perspective. Soc. Econ. Paleontol. Mineral., Spec. Publ., 32:181-207.

Graham, D. W., Corliss, B. H., Bender, M. L., and Keigwin, L. D., Jr., 1981. Carbon and oxygen isotopic disequilibria of recent deep-sea benthic foraminifera. Mar. Micropaleontol., 6:483-497.

Haq, B. U., Premoli Silva, I., and Lohman, G. P., 1977. Calcareous plankton paleobiogeographic evidence for major climatic fluctuations in the early Cenozoic Atlantic Ocean. J. Geophys. Res., 82: 3861-3876.

Hsü, K. J., McKenzie, J. A., Oberhänsli, H., Weissert, H., and Wright, R. C., 1984. South Atlantic Cenozoic paleoceanography. In Hsü, K. J., LaBrecque, J. L., et al., Init. Repts. DSDP, 73: Washington (U.S. Govt. Printing Office), 771-785.

Katz, M. E., and Miller, K. G., 1991. Early Paleogene benthic foraminiferal assemblage and stable isotope composition in the Southern Ocean, Ocean Drilling Program, Leg 114. In Ciesielski, P. F., Kristoffersen, Y., et al., Proc. ODP, Sci. Results, 114: College Station, TX (Ocean Drilling Program).

Keigwin, L., and Keller, G., 1984. Middle Oligocene climate change from equatorial Pacific Site 77. Geology, 12:16-19.

Keigwin, L. D., and Corliss, B. H., 1986. Stable isotopes in late middle Eocene to Oligocene forams. Geol. Soc. Am. Bull., 97:335-345.

Keller, G., Herbert, T., Dorsey, R., D'Hondt, S., Johnsson, M., and Chi, W. R., 1987. Global distribution of late Paleogene hiatuses. Geology, 15:199-203.

Kemp, E. M., 1978. Tertiary climatic evolution and vegetation history in the southeast Indian Ocean region. Palaeogeogr., Palaeoclimatol., Palaeoecol., 24:169-208.

Kennett, J. P., 1977. Cenozoic evolution of Antarctic glaciation, the circum-Antarctic Ocean, and their impact on global paleoceanography. J. Geophys. Res., 82:3843-3860.

Kennett, J. P., and Shackleton, N. J., 1976. Oxygen isotopic evidence for the development of the psychrosphere $38 \mathrm{Myr}$ ago. Nature, 260: 513-515.
Kennett, J. P., and Stott, L. D., 1990. Proteus and proto-oceanus: ancestral paleogene oceans as revealed from Antarctic stable isotopic results: ODP Leg 113. In Barker, P. F., Kennett, J. P., et al., Proc. ODP, Sci. Results, 113: College Station, TX (Ocean Drilling Program), 865-880.

Kennett, J. P., Burns, R. E., Andrews, J. E., Churkin, M., Jr., Davies, T. A., Dumitrica, P., Edwards, A. R., Galehouse, J. S., Packham, G. H., and van der Lingen, G. J., 1972. Australian-Antarctic continental drift, paleo-circulation change and Oligocene deep-sea erosion. Nature, 239:51-55.

La Brecque, J. L., and Rabinowitz, P. D., 1977. Magnetic anomalies bordering the continental margin of Argentina. AAPG, Spec. Map Ser., Cat. 826.

Luyendyk, B. P., and Davies, T. A., 1974. Results of DSDP Leg 26 and the geologic history of the Southern Indian Ocean. In Davies, T. A., Luyendyk, B. P., et al., Init. Repts. DSDP, 26: Washington (U.S. Govt. Printing Office), 909-952.

Matthews, R. K., and Poore, R. Z., 1980. Tertiary $\delta^{18} \mathrm{O}$ record and glacio-eustatic sea-level fluctuations. Geology, 8:501-504.

McKenzie, D. P., and Sclater, J. G., 1971. The evolution of the Indian Ocean since the late Cretaceous. Geophys. J. R. Astron. Soc., 25: 437-528.

Miller, K. G., and Fairbanks, R. G., 1983. Evidence for Oligocene-middle Miocene abyssal circulation changes in the western North Atlantic. Nature, 306:250-253.

Miller, K. G., Fairbanks, R. G., and Mountain, G. S., 1987. Tertiary oxygen isotope synthesis, sea-level history, and continental margin erosion. Paleoceanography, 2:1-19.

Miller, K. G., Feigson, M. D., and Olsson, R. K., 1988. Upper Eocene to Oligocene isotope $\left.{ }^{87} \mathrm{Sr} /{ }^{86} \mathrm{Sr}, \delta^{18} \mathrm{O}, \delta^{13} \mathrm{C}\right)$ standard section, Deep Sea Drilling Project Site 522. Paleoceanography, 3:223-233.

Miller, K. G., Janecek, T. R., Katz, M. E., and Keil, D. J., 1987. Abyssal circulation and benthic foraminiferal changes near the Paleocene/Eocene boundary. Paleoceanography, 2:741-761.

Miller, K. G., and Thomas, E., 1985. Late Eocene to Oligocene benthic foraminiferal isotopic record, Site 574, equatorial Pacific. In Mayer, L., Theyer, F., et al., Init. Repts. DSDP, 85: Washington (U.S. Govt. Printing Office), 771-777.

Miller, K. G., and Tucholke, B. E., 1983. Development of Cenozoic abyssal circulation south of the Greenland-Scotland Ridge. In Bott, M., Saxov, S., Talwani, M., and Theide, J. (Eds.), Structure and Development of the Greenland-Scotland Ridge: New York (Plenum Publ.), 549-589.

Moore, T. C., Jr., Rabinowitz, P. D., Borella, P. E., Shackleton, N. J., and Boersma, A., 1984. History of the Walvis Ridge. In Moore, T. C., Rabinovitz, P. D., et al., Init. Rept. DSDP, 74: Washington (U.S. Govt. Printing Office), 873-894.

Oberhansli, H., 1986. Latest Cretaceous-Early Neogene oxygen and carbon isotopic record at DSDP sites in the Indian Ocean. Mar. Micropaleontol., 10:91-117.

Oberhansli, H., McKenzie, J. A., Toumarkine, M., and Weissert, H., 1984. A paleoclimatic and paleoceanographic record of the Paleogene in the central South Atlantic (Leg 73, Sites 522, 523, and 524). In Hsü, K. J., LaBrecque, J. L., et al., Init. Repts. DSDP, 73: Washington (U.S. Govt. Printing Office), 737-747.

Oberhansli, H., and Toumarkine, M., 1985. The Paleogene oxygen and carbon isotope history of Sites 522,523 , and 524 from the central South Atlantic. In Hsü, K. J., and Weissert, A. (Eds.), South Atlantic Paleoceanography: Cambridge (Cambridge Univ. Press), 124-147.

O'Neil, J. R., Clayton, R. N., and Mayeda, T. K., 1969. Oxygen isotope fractionation in divalent metal carbonates. J. Chem. Phys., 51: $5547-5558$

Ostlund, H. G., Craig, C., Broecker, W. S., and Spencer, D. (Eds.), 1987. Geosecs Atlantic, Pacific, and Indian Ocean expeditions. Shorebased data and graphics. Geosecs Atlas Series (Vol. 7): Washington (U.S. Govt. Printing Office).

Owens, R. E., and Rea, D. K., 1985. Sea-floor hydrothermal activity links climate to tectonics: the Eocene $\mathrm{CO}_{2}$ greenhouse. Science, 227: 166-169.

Poore, R. Z., and Matthews, R. K., 1984. Oxygen isotope ranking of late Eocene and Oligocene planktonic foraminifers: implications for Oligocene sea-surface temperatures and global ice-volume. Mar. Micropaleontol., 9:111-134. 
Sancetta, C. A., 1979. Paleogene Pacific microfossils and paleoceanography. Mar. Micropaleontol., 4:363-398.

Savin, S. M., Abel, L., Barrera, E., Hodell, D., Kennett, J. P., Murphy, M., Keller, G., Killingley, J., and Vincent, E., 1985. The evolution of Miocene surface and near-surface marine temperatures: oxygen isotopic evidence. In Kennett, J. P. (Ed.), The Miocene Ocean: Paleoceanography and Biogeography: Geol. Soc. Am. Spec. Publ., 163: 49-82.

Savin, S. M., Douglas, R. G., Keller, G., Killingley, J. S., Shaughenssy, L., Sommer, M. A., Vincent, E., and Woodruff, F., 1981. Miocene benthic foraminiferal isotope records: a synthesis. Mar. Micropaleontol., 6:423-450.

Savin, S. M., Douglas, R. G., and Stehli, F. G., 1975. Tertiary marine paleotemperatures. Geol. Soc. Am. Bull., 86:1499-1510.

Sclater, J. G., Abbot, D., and Thiede, J., 1977. Paleobathymetry and sediments of the Indian Ocean. In Heirtzler, J., Bolli, H. M., Davies, T. A., Saunders, J. B., and Sclater, J. G. (Eds.), Indian Ocean Geology and Biostratigraphy: Am. Geophys. Union, 25-59.

Shackleton, N. J., 1974. Attainment of isotopic equilibrium between ocean water and benthonic foraminifera genus Uvigerina: isotopic changes in the ocean during the last glacial. Les Methodes Quantitative d'etude des Variations due Climat au Cours du Pleistocene, Colloques Internationaux due C.N.R.S., 219:203-209.

1986. Paleogene stable isotope events. Palaeogeogr., Palaeoclimat., Palaeoecol., 57:91-102.

1987. The carbon isotope record of the Cenozoic: history of organic carbon burial and of oxygen in the ocean and atmosphere. In Brooks, J., and Fleet, A. J. (Eds.), Marine Petroleum Source Rocks. Geol. Soc. Spec. Publ. London, 26:423-434.

Shackleton, N. J., and Boersma, A., 1981. The climate of the Eocene ocean. J. Geol. Soc. London, 138:153-157.

Shackleton, N. J., Corfield, R. M., and Hall, M. A., 1985. Stable isotope data and the ontogeny of Paleocene planktonic foraminifera. $J$. Foraminiferal Res., 15:321-336.

Shackleton, N. J., and Kennett, J. P., 1975. Paleotemperature history of the Cenozoic and the initiation of Antarctic glaciation: oxygen and carbon isotope analyses in DSDP Sites 277, 279 and 281. In Kennett,
J. P., Houtz, R. E., et al., Init. Repts. DSDP, 29: Washington (U.S. Govt. Printing Office), 743-755.

Shackleton, N. J., and Hall, M. A., 1984. Carbon isotope data from Leg 74 sediments. In Moore, T. C., Jr., Rabinowitz, P. D., et al., Init. Repts. DSDP, 74: Washington (U.S. Govt. Printing Office), 613-619.

Shackleton, N. J., Hall, M. A., and Boersma, A., 1984. Oxygen and carbon isotope data from Leg 74 foraminifers. In Moore, T. C., Jr. Rabinowitz, P. D., et al., Init. Repts. DSDP, 74: Washington (U.S. Govt. Printing Office), 599-612.

Stott, L. D., Kennett, J. P., Shackleton, N. J., Corfield, R. M., 1990. The evolution of Antarctic surface waters during the Paleogene: inferences from stable isotopic composition of planktonic foraminifers, ODP Leg 113. In Barker, P. F., Kennett, J. P., et al., Proc. ODP, Sci. Results, 113: College Station, TX (Ocean Drilling Program), 849-863.

van Andel, T. H., Thiede, J., Sclater, J. G., and Hay, W. W., 1977. Depositional history of the South Atlantic Ocean during the last 125 million years. J. Geol., 85:651-698.

Williams, D. F., Bé, A.W.H., and Fairbanks, R. G., 1979. Seasonal oxygen isotopic variations in living plantonic foraminifera off Bermuda. Science, 206:447-449.

Williams, D. F., Sommer, M. A., II, and Bender, M. L., 1977. Carbon isotopic compositions of Recent planktonic foraminifers of the Indian Ocean. Earth Planet. Sci. Lett., 36:391-403.

Wolfe, J. A., 1978. A paleobotanical interpretation of Tertiary climates in the Northern Hemisphere. Am. Sci., 66:694-703.

Woodruff, F., and Savin, S., 1989. Miocene deepwater oceanography. Paleoceanography, 4:87-140.

Woodruff, F., Savin, S. M., and Douglas, R. G., 1980. Biological fractionation of oxygen and carbon isotopes by Recent benthic foraminifera. Mar. Micropaleontol., 5:3-11.

Date of initial receipt: 19 March 1990

Date of acceptance: 8 August 1990

Ms 119B-167 